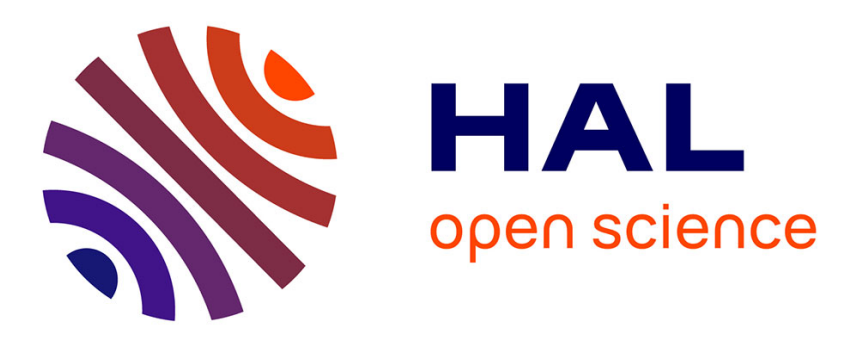

\title{
Long-range ordered and atomic-scale control of graphene hybridization by photocycloaddition
}

Miao Yu, Chong Chen, Qi Liu, Cristina Mattioli, Hongqian Sang, Guoqiang Shi, Wujun Huang, Kongchao Shen, Zhuo Li, Pengcheng Ding, et al.

\section{- To cite this version:}

Miao Yu, Chong Chen, Qi Liu, Cristina Mattioli, Hongqian Sang, et al.. Long-range ordered and atomic-scale control of graphene hybridization by photocycloaddition. Nature Chemistry, 2020, 12 (11), pp.1035-1041. 10.1038/s41557-020-0540-2 . hal-03064896

\section{HAL Id: hal-03064896 https://hal.science/hal-03064896}

Submitted on 14 Dec 2020

HAL is a multi-disciplinary open access archive for the deposit and dissemination of scientific research documents, whether they are published or not. The documents may come from teaching and research institutions in France or abroad, or from public or private research centers.
L'archive ouverte pluridisciplinaire HAL, est destinée au dépôt et à la diffusion de documents scientifiques de niveau recherche, publiés ou non, émanant des établissements d'enseignement et de recherche français ou étrangers, des laboratoires publics ou privés. 


\title{
Long-range ordered and atomic-scale control of graphene hybridization by photocycloaddition
}

\author{
Miao $\mathrm{Yu}^{1, \#, *}$, Chong Chen ${ }^{1, \#}$, Qi Liu ${ }^{3, \#}$, Cristina Mattioli ${ }^{2}$, Hongqian Sang ${ }^{4,5}$, Guoqiang Shi ${ }^{1}$, Wujun \\ Huang ${ }^{6}$, Kongchao Shen ${ }^{7}$, Zhuo $\mathrm{Li}^{1}$, Pengcheng Ding ${ }^{8}$, Pengfei Guan ${ }^{3}$, Shaoshan Wang ${ }^{1}$, Ye Sun ${ }^{8}$, \\ Jinping $\mathrm{Hu}^{7}$, André Gourdon ${ }^{2, *}$, Lev Kantorovich ${ }^{4, *}$, Flemming Besenbacher ${ }^{9, *}$, Mingshu Chen ${ }^{6}$, Fei \\ Song ${ }^{7}$, and Federico Rosei ${ }^{10}$
}

1. State Key Laboratory of Urban Water Resource and Environment, School of Chemistry and Chemical Engineering, Harbin Institute of Technology, Harbin 150001, China

2. CEMES-CNRS, Toulouse 31055, France

3. Beijing Computational Science Research Center, Beijing 100193, China

4. Department of Physics, King's College London, The Strand, London WC2R 2LS, U.K.

5. Institute for Interdisciplinary Research, Jianghan University, Wuhan 430056, China

6. Department of Chemistry, Xiamen University, Xiamen 361005, China

7. Shanghai Synchrotron Radiation Facility, Shanghai Advanced Research Institute, Chinese Academy of Sciences, Shanghai 201200, China

8. Condensed Matter Science and Technology Institute, Harbin Institute of Technology, Harbin 150001, China

9. iNANO and Department of Physics and Astronomy, Aarhus University, Aarhus 8000, Denmark

10. INRS Centre for Energy, Materials and Telecommunications, Varennes J3X 1S2, Canada

\# These authors contributed equally to this work.

Correspondence to: miaoyu_che@hit.edu.cn, andre.gourdon@cemes.fr, lev.kantorovitch@kcl.ac.uk, and fbe@inano.au.dk 


\begin{abstract}
Chemical reactions converting $s p^{2}$ to $s p^{3}$ hybridization have been demonstrated to be a fascinating yet challenging route to functionalize graphene. So far, it has not been possible to precisely control the reaction sites nor their lateral order at the atomic/molecular scale. The application prospects have been limited for reactions requiring long soaking, heating, electric pulses, or probe tip press. Herein, we demonstrate a spatially-selective photocycloaddition reaction of a two-dimensional (2D) molecular network with defect-free basal plane of single-layer graphene. Directly visualized at the sub-molecular level, the cycloaddition is triggered by ultraviolet irradiation in ultrahigh vacuum, requiring no aid of the graphene Moiré pattern. The reaction involves both $[2+2]$ and $[2+4]$ cycloaddition, with the reaction sites aligned into a 2D extended and well-ordered array, inducing a bandgap for the reacted graphene layer. This work provides a solid base for designing and engineering graphene-based optoelectronic and microelectronic devices.
\end{abstract}


Since its isolation as a two dimensional (2D) system, graphene has been widely acclaimed due to its extraordinary physical and chemical properties, showing great promise for next-generation electronic and optoelectronic devices ${ }^{1-3}$. However, graphene's zero bandgap limits its use as an active layer in electronic devices. To pave the way towards practical applications, the functionalization of graphene has been a topic of much interest ${ }^{4}$. A broad variety of functionalization approaches have been developed, such as doping with heteroatoms $s^{5,6}$, decoration with nanoparticles ${ }^{7}$, fabrication of nanostructured graphene ${ }^{8-18}$, as well as chemical reactions with atomic hydrogen, fluorine, and other reactants ${ }^{19-26}$. Despite significant progress, controllable and precise functionalization of graphene remains a major challenge.

Cycloaddition has been demonstrated to be an effective path for introducing $s p^{3}$ hybridization and thereby functionalizing graphene ${ }^{26-28}$. Confined by the pi-conjugation form of the carbon atoms, covalent addition on graphene's basal plane needs to overcome a large energy barrier. The reactions therefore take place preferentially at graphene defects and edges, and have a low probability of occurring on defect-free pristine graphene planes ${ }^{29}$. Even if the reactions were induced, they occur randomly ${ }^{4}$ or strongly depend on the graphene Moiré pattern formed on specific substrates ${ }^{30,31}$, and it is difficult to exclude the disturbance from solvent and impurities for the reactions occurred in solution. So far, it has not been possible to realize chemical reactions of graphene with molecular networks or to achieve 2D order of reaction sites with atomic/molecular precision. Superior to the reactions requiring long soaking, heating, electric pulse, or probe tip press, the merits of photocycloaddition, such as simplicity, remote controllability, and compatibility with other photorelated techniques, have been recognized; nevertheless, photocycloaddition of graphene has been scarcely reported, with only precedent in aqueous solution ${ }^{32}$.

Here, we report an in situ photocycloaddition reaction of an extended molecular network placed on a single-layer defect-free pristine graphene sheet in ultrahigh vacuum (UHV), directly demonstrated by high-resolution scanning tunnelling microscopy (STM), infrared reflection- 
absorption spectroscopy (IRAS), angle resolved photoemission spectroscopy (ARPES), Raman spectroscopy, and confirmed by ab initio density functional theory (DFT) calculations. The molecule used is maleimide derivative (Fig. 1), i.e. 3,5-bis(carboxylic acid)-phenyl-3-maleimide $\left(\mathrm{C}_{12} \mathrm{H}_{7} \mathrm{NO}_{6}\right.$, denoted as BCM), which undergoes a cycloaddition reaction with graphene. The carboxylic acid groups in the meta-position can polarize the $\mathrm{C}=\mathrm{C}$ of the maleimide and hence enhance its reactivity with the surface ${ }^{26,33}$, meanwhile enable intermolecular hydrogen bonding. The reaction is triggered by ultraviolet (UV) irradiation at room temperature (RT), with the reaction sites being spatially selective and arranged in 2D long-range order.

\section{Results and discussion}

Since a graphene layer alone is rather 'slippery' for adsorbed organic molecules (as the latter normally prefer accommodating on metal surfaces than on graphene $)^{34}$, an extended flake of graphene, rather than small patches, is crucial for the formation of a well-defined molecular network on a graphene-metal substrate. In this work, a single-layer graphene sheet was epitaxially grown on $\mathrm{Cu}(111)$ by chemical vapor deposition with ethyne as the precursor [see Supporting Information (SI)]. A defect-free full layer of graphene (Figs. 2a-b) can be obtained at $993 \mathrm{~K}$ upon only one cycle of 20min growth.

When BCM molecules are thermally sublimated from a crucible and adsorbed on a single-layer graphene sheet grown on $\mathrm{Cu}(111)$ kept at RT, a well-ordered 2D network forms spontaneously on the surface. At high doses, the network can cover the entire surface (Supplementary Fig. 1), showing neither corrugation nor variations associated with the graphene Moiré pattern. Each individual molecule is imaged as two sub-protrusions, one circular and one triangular (Fig. 2c), attributed to the maleimide group and the bis(carboxylic acid)-phenyl group, respectively. Upon closer inspection, the network is found to be tiled by the hexapetalous flowers as building blocks (Fig. 2d). Each flower is composed of six molecules, and the flowers are closely crossed in three equivalent directions into the extended network, with periodicity $a=b=26.5 \pm 0.5 \AA$ and angle between the two vectors of $\sim 60^{\circ}$. Relative to the graphene layer, the deviation angle of the network is $\sim 11^{\circ}$.

We performed DFT calculations to understand the formation of the BCM network on the graphene-Cu(111) substrate. The calculated unit cell involved six BCM molecules, one single graphene layer, and three layers of Cu substrate. As illustrated in Fig. 2e, the graphene layer is parallel to the $\mathrm{Cu}(111)$ surface, and all molecules are physisorbed on the graphene layer in a flat geometry at a vertical BCM-graphene distance of $3.4 \AA$, showing no significant charge transfer with the graphene-Cu(111) substrate (Supplementary Fig. 2). Within each hexapetalous flower, the molecules are associated with intermolecular double $\mathrm{O}-\mathrm{H} \cdots \mathrm{O}$ hydrogen bonds between the carboxylic acid groups of the neighboring molecules, resulting in a stabilization energy of -1.01 
eV/molecule (Fig. 2f). The characteristic 'kebab' charge distribution ${ }^{35}$ associated with hydrogen bonds of each BCM is shown in Supplementary Fig. 3. The calculated network has a periodicity of $26.1 \AA$ and is rotated by $10.9^{\circ}$ relative to the underlying pristine graphene (Fig. $2 \mathrm{~g}$ and Supplementary Fig. 4), in good agreement with the experimental results (Fig. 2h). Both experimental observations and the simulated structure (Fig. 2i) reveal nearly identical tunneling contrast for all molecules.

When the BCM layer on graphene-Cu(111) is exposed to UV irradiation (centered at $365 \mathrm{~nm}$ with a power density of $40 \mathrm{~mW} \cdot \mathrm{cm}^{-2}$, covering the entire sample surface) for $8 \mathrm{~min}$, the tunneling contrast of molecules at certain sites of the BCM network changes, while the arrangement of the network remains the same (Figs. 3a-b and Supplementary Fig. 5). Four molecules (denoted as ' $\alpha$ ') at specific positions in each unit cell appear to be darker than the other two molecules (' $\beta$ '). While $\beta$ maintain almost the same contrast and morphology as before irradiation, each $\alpha$ has a varied morphology besides its lower brightness, presenting a slightly distorted protrusion corresponding to the bis(carboxylic acid)-phenyl group and a less visible circular lobe of the maleimide group.

The variation triggered by UV irradiation was further explored using DFT calculations. The observed distinction between $\alpha$ and $\beta$ is found to result from the cycloaddition of the BCM network with the graphene layer. To achieve this cycloaddition state upon DFT relaxation, all six molecules of each unit cell are initially prepared with their maleimide groups being bent towards the graphene layer, as otherwise the ground state (i.e. all BCMs being flat) would be attained. We tried a number of models by varying the maleimide bending degree ( $30-40^{\circ}$ relative to the horizon): in all cases, although all the molecules are bent in the same way in each model and their $\mathrm{C}=\mathrm{C}$ groups have the same initial distance to the graphene plane (2.0-2.3 $\AA$ ), only four molecules further relax into the bent geometry whilst the other two restore the flat form after a full relaxation.

The resultant configuration is presented in Figs. 3c and 3d: four molecules $(\alpha)$ in each unit cell are covalently bonded to the graphene layer; the other two $(\beta)$ lie flat, remaining physisorbed. The bending degree of the maleimide ring is $34^{\circ}$ for $\alpha 1$ and $\bar{\alpha} 1$, and $30^{\circ}$ for $\alpha 2$ and $\bar{\alpha} 2$; the bond length between each carbon atom in the opened $\mathrm{C}=\mathrm{C}$ and its bonded carbon atom in the graphene layer is $1.63 \AA$ for $\alpha 1$ and $\bar{\alpha} 1$, and $1.48 \AA$ for $\alpha 2$ and $\bar{\alpha} 2$. Even though an energy penalty is imposed by the maleimide bending upon the cycloaddition, the bis(carboxylic acid)-phenyl group of each reacted BCM remains nearly flatly lying on the surface rather than standing up, due to the interaction with the graphene- $\mathrm{Cu}(111)$ substrate and laterally intermolecular double HB. The average interaction between a single $\alpha$ and graphene is substantially significant ( $-2.09 \mathrm{eV}$ lower), showing typical covalent bonding. The overall energy (per unit) after irradiation is by $8.26 \mathrm{eV}$ less negative than that of the unreacted system (Supplementary Table 1), suggesting that the reaction is highly endothermic. 
The spatial selectivity of the reaction sites originates from the geometry of the $\mathrm{BCM}$ network relative to the underlying graphene. As depicted by Figs. $3 d$ and 3e, the relative $\mathbf{B C M}$ layer-graphene geometry allows the $\mathrm{C}=\mathrm{C}$ in the maleimide group of only $\alpha$ (four molecules out of six in each unit) to have an appropriate angle and distance for covalent bonding with $\mathrm{C}$ atoms of the graphene layer. Cycloaddition reaction with graphene involves different types ${ }^{23,26}: C 2, C 3$ and $C 4$ denote the cycloaddition with $(1,2),(1,3)$ and $(1,4)$ carbon atoms of a graphene hexagon, respectively. Both $[2+2]$ $(C 2)$ and $[2+4](C 4)$ cycloaddition participate in this present reaction with two $C 2$ and two $C 4$ for each unit; the relative $\mathbf{B C M}$-graphene geometry prevents $C 3$ cycloaddition. Such combination of $C 2+C 4$ on graphene- $\mathrm{Cu}(111)$ is distinct from the reported single $\mathrm{C} 2$ cycloaddition for graphene on $\mathrm{SiC}^{26}$, or $\mathrm{C3}$ for graphene on iridium ${ }^{23}$. The calculated STM image based on the structural model of this spatiallyselective cycloaddition provides a good match for the experimental result (Fig. 3f).

The occurrence of the photocycloaddition reaction between the BCM layer and graphene was further verified using IRAS (Fig. 4a and Supplementary Fig. 6). Due to the flat to non-flat structural transition upon the cycloaddition reaction, the vibrations at $829 \mathrm{~cm}^{-1}$ and $1609 \mathrm{~cm}^{-1}$, corresponding to the out-of-plane $\mathrm{C}-\mathrm{H}$ bending vibration of maleimide and the aromatic $\mathrm{C}-\mathrm{C}$ stretching vibration, are significantly enhanced after the reaction. Meanwhile, newly appearing peaks, i.e. a pronounced one at $1509 \mathrm{~cm}^{-1}$ and two very weak ones at $864 \mathrm{~cm}^{-1}$ and $1039 \mathrm{~cm}^{-1}$, are observed. Based on DFT and density functional perturbation theory (DFPT) calculations, these new peaks are originated from the $C-C / C=C$ stretching vibrations of the $[2+2]$ and $[2+4]$ ring (Fig. $4 b$ and Supplementary Fig. 7 ), further confirming the coexistence of $C 2$ and $C 4$ cycloaddition.

The cycloaddition was also evidenced by the results of Raman spectroscopy for quasi-freestanding graphene on mica (Supplementary Fig. 8). While the Raman spectrum shows almost no variation from the case of pristine graphene after depositing the BCM layer and prior to UV irradiation, the relative intensity of the D-band to the G-band $\left(I_{D} / I_{G}\right)$ is evidently enhanced by the irradiation, due to the conversion of $s p^{2}$ to $s p^{3}$ hybridization at the reaction sites. The results are in good agreement with earlier Raman results of graphene covalently-bonded with molecules ${ }^{27,36}$.

The variation of graphene's band structure upon photocycloaddition was explored using ARPES. The data sets were recorded as 3D maps with the photoemission intensity measured as a function of binding energy and momentum space ( $\mathrm{k}$ vector) parallel to the surface. Distinct from the linear crossing of the typical Dirac band for pristine graphene (Figs. 4c-d), a small gap and parabolic dispersion are evident for the reacted graphene (Fig. 4e), consistent with previous work on covalently grafted graphene ${ }^{26}$. Similar to the reported bandgap opening in functionalized graphene sheets ${ }^{20}$, we assume a symmetric opening. By extracting energy distribution curves from the ARPES data and fitting them with Gaussian function, the value of the band gap is estimated as 170 meV. 
In addition, the boosted stability of the irradiated BCM layer demonstrates its covalent bonding with graphene as well. The molecular layer is relatively fragile before irradiation (Supplementary Fig. 9), and STM scanning at RT can not only ruin it and enlarge the defect areas (marked by the green rectangles), but also reorganize the molecules and restore the order of the network (marked by the blue rectangles). After irradiation, under nearly identical scanning conditions, the network is rather robust upon RT scanning, remaining unchanged over a long-term repeated scanning. Moreover, complete desorption is achieved at $\sim 376 \mathrm{~K}$ for the non-irradiated case. In contrast, after annealing at the same temperature for $10 \mathrm{~min}, \sim 75 \%$ of the irradiated BCM layer is preserved and remains wellordered without noticeable molecular rearrangement compared with that before annealing (Supplementary Fig. 10), indicating the reaction yield and the high thermodynamic stability of the product. Consistently, Climbing Image Nudged Elastic Band (CI-NEB) calculations show that a significant barrier is required to break the covalent bonds, rendering the excited configuration sufficiently stable (Supplementary Fig. 11).

Besides the experimental evidence, the feasibility of triggering the cycloaddition between BCM and graphene upon the applied photoexcitation is further evaluated by DFT calculations. We calculated the energy increase $(\Delta E)$ from the initial (physisorbed) to the final (covalently-bonded) state of a single BCM cycloaddition with graphene (Supplementary Table 2). For free-standing graphene, $\Delta E$ for $C 2$ and $C 4$ is 2.18 and $2.11 \mathrm{eV}$, respectively. When the $\mathrm{Cu}(111)$ substrate is included, a small increase of $\Delta E$ is found for both $C 2$ and $C 4$. The Fermi energy of graphene is slightly shifted due to electron injection from the Cu substrate ${ }^{37}$. Various relative positions of graphene on $\mathrm{Cu}(111)$ (Supplementary Fig. 12) are considered to simulate different graphene Moiré patterns. In all cases, $\Delta E \leq 2.24 \mathrm{eV}$, which can be satisfied by the applied irradiation (centered at $\sim 3.4 \mathrm{eV}$ ). Using timedependent DFT (TD-DFT) simulations, we further calculated the excitation energies of the vertical singlet-to-singlet transitions for a single BCM molecule in $C 4$ cycloaddition with free-standing graphene (Supplementary Figs. 13-14). As summarized in Supplementary Table 3, significant oscillator strengths are obtained at transition energies of $\sim 2.8 \mathrm{eV}$, which is also satisfied by the photon energy used in the experiments. As the formation of the non-irradiated molecular adlayer is not correlated with the graphene Moiré patterns (Figs. 2c-e and Supplementary Fig. 1) and since our Raman results indicate that BCM can graft on quasi-free-standing graphene (on mica) in addition to graphene- $\mathrm{Cu}(111)$, the observed reaction does not require the aid of graphene Moiré patterns. Such independence is beneficial for electronic applications, and distinct from the former grafting of phthalocyanines on $\operatorname{Ir}(111)^{23}$ or cyanomethyl radicals on $\mathrm{Ru}(0001)^{30,31}$ where the aid of graphene Moiré patterns is indispensable. 
Besides providing photons with an average energy of $3.4 \mathrm{eV}, \mathrm{UV}$ irradiation also increases the sample temperature, in an irradiation-duration dependent fashion, e.g. the sample temperature rises to $\sim 312 \mathrm{~K}$ after $8 \mathrm{~min}$ and to $\sim 319 \mathrm{~K}$ after $25 \mathrm{~min}$ of irradiation (Supplementary Fig. 15). Control experiments reveal that neither annealing alone up to $360 \mathrm{~K}$ nor irradiation without temperature increase can trigger the reaction, suggesting that both the photon excitation and enhanced thermal vibration play a crucial role. Indeed, when the system is taken into a high-lying excited adiabatic potential energy surface (PES) by the photons, it then experiences a cascade of transitions from that PES to certain lower lying PESs until the system reaches the new stable state (the reaction product). The ionic relaxation of the system along this process results in a temperature increase due to the released phonons, and benefits overcoming energy barriers that appear along the relaxation path and eventually achieving the final reaction product.

Cycloaddition is known as a reversible reaction in solution ${ }^{27}$ : under appropriate conditions, bidirectional reactions (' $A$ ' $=>{ }^{\prime} B$ ' and ' $B$ ' $=>^{\prime} A$ ', where ' $A$ ' and ' $B$ ' represent the unreacted and reacted state) occur synchronously. Intriguingly, in the present case, besides the occurrence of ' $A$ ' $=>$ ' $B$ ', the tendency of ' $B$ ' =>'A' is observed after applying another round of UV irradiation with extended exposure (e.g. $11 \mathrm{~min}$ ): annealing the twice-irradiated sample at $\sim 376 \mathrm{~K}$ for $10 \mathrm{~min}$ results in a much lower coverage of BCM layer compared with that after the first-round irradiation ( $<5 \%$ vs. $~ 75 \%$ ); the peaks corresponding to vibrations due to the covalent bonding in IRAS and $I_{D} / I_{G}$ in Raman spectrum all largely decrease (Fig. 4b and Supplementary Fig. 6); the tunnelling contrast and morphology of most BCM domains restore to those without UV irradiation. Nevertheless, upon a sufficiently long exposure to irradiation (e.g. $30 \mathrm{~min}$ ), the reaction yield fixes at $\sim 56 \%$, which does not change upon a longer irradiation (e.g. $60 \mathrm{~min})$.

A plausible explanation is as follows: (1) the actual reaction path for ' $B{ }^{\prime}=>^{\prime} A$ ' is distinct from that of ' $A$ ' $=>$ ' $B$ ' in its backward direction: a higher temperature is required to overcome the energy barriers for the reverse reaction, hence only the forward reaction occurs upon the first irradiation for $8 \mathrm{~min}$; the ' $A$ ' =>'B' yield is reduced when the irradiation is extended (e.g. $11 \mathrm{~min}, 30 \mathrm{~min}$ ) because the further increased sample temperature renders the reverse reaction more probable; (2) upon a short irradiation duration (e.g. 8 or $11 \mathrm{~min}$ ), the system is in a dynamic intermediate state out of equilibrium and shows dominantly ' $\mathrm{B}$ ' or ' $\mathrm{A}$ ' ; while upon a sufficiently long irradiation (e.g. 30 or 60 min), molecules can flip between the unreacted (flat) and reacted (bonded) states all the time due to sufficient photo- and thermal excitation and reach an equilibrium state with a steady reaction yield. Based on our findings, it remains a challenge to uncover the actual reaction process and the exact role of the irradiation-induced temperature elevation for the reversible reaction. A comprehensive 
investigation on the reversibility of photocycloaddition would be appropriate for a future study, to fully understand the underlying intricacies.

\section{Conclusions}

Employing a specially designed BCM molecule equipped with maleimide and dicarboxylic acid groups, we have demonstrated a cycloaddition between an extended molecular network with defectfree pristine graphene basal plane in UHV. The reaction involves both [2+2] and $[2+4]$ cycloaddition, requiring no aid of the graphene Moiré pattern. The reaction sites are spatially selective and aligned into a 2D ordered array. Such well-defined tuning of graphene's electronic structure may hold great promise for cutting-edge microelectronic devices, e.g. atomic-scale circuits. The photocycloaddition pathway not only provides a practical solution for the highly endothermic reaction, but also is favourable for applications in optoelectronic devices with distinct advantages, such as simplicity, remote controllability, and potential compatibility with the widely-used photo-related techniques, e.g. photolithography. As the first demonstration for the reaction of graphene with a molecular network, this work unlocks an efficient and convenient route to fabricate a stable, highly precise and long-range ordered electronic matrix of graphene, and may spark considerable enthusiasm in achieving well-controlled functionalization of graphene by facile photo-induced chemical reactions with supramolecular networks for designing and engineering graphene-based optoelectronic and nanoelectronic devices.

\section{References}

1. Geim, A. K. Graphene: status and prospects. Science 324, 1530-1534 (2009).

2. Patera, L. L. et al. Real-time imaging of adatom-promoted graphene growth on nickel. Science 359, 1243-1246 (2018).

3. Schwierz, F. Graphene transistors. Nat. Nanotech. 5, 487-496 (2010).

4. Yan, L. et al. Chemistry and physics of a single atomic layer: strategies and challenges for functionalization of graphene and graphene-based materials. Chem. Soc. Rev. 41, 97-114 (2012).

5. Zhao, L. Y. et al. Visualizing individual nitrogen dopants in monolayer graphene. Science 333, 999-1003 (2011).

6. Ci, L. J. et al. Atomic layers of hybridized boron nitride and graphene domains. Nat. Mater. 9, 430-435 (2010).

7. Nair, M. N. et al. High van Hove singularity extension and Fermi velocity increase in epitaxial graphene functionalized by intercalated gold clusters. Phys. Rev. B 85, 245421-245426 (2012).

8. Bai, J. W. et al. Graphene nanomesh. Nat. Nanotech. 5, 190-194 (2010). 
9. Treier, M. et al. Surface-assisted cyclodehydrogenation provides a synthetic route towards easily processable and chemically tailored nanographenes. Nat. Chem. 3, 61-67 (2011).

10. Palma, C. A. et al. Photoinduced $\mathrm{C}-\mathrm{C}$ reactions on insulators toward photolithography of graphene nanoarchitectures. J. Am. Chem. Soc. 136, 4651-4658 (2014).

11. Narita, A. et al. Synthesis of structurally well-defined and liquid-phase-processable graphene nanoribbons. Nat. Chem. 6, 126-132 (2014).

12. Cai, J. M. et al. Graphene nanoribbon heterojunctions. Nat. Nanotech. 9, 896-900 (2014).

13. Chen, Y. C. et al. Molecular bandgap engineering of bottom-up synthesized graphene nanoribbon heterojunctions. Nat. Nanotech. 10, 156-160 (2015).

14. Han, P. et al. Self-assembly strategy for fabricating connected graphene nanoribbons. ACS Nano 9, 12035-12044 (2015).

15. Ruffieux, P. et al. On-surface synthesis of graphene nanoribbons with zigzag edge topology. Nature 531, 489-492 (2016).

16. Chen, Z. P. et al. Synthesis of graphene nanoribbons by ambient-pressure chemical vapor deposition and device integration. J. Am. Chem. Soc. 138, 15488-15496 (2016).

17. Nguyen, G. D. et al. Atomically precise graphene nanoribbon heterojunctions from a single molecular precursor. Nat. Nanotech. 12, 1077-1082 (2017).

18. Moreno, C. et al. Bottom-up synthesis of multifunctional nanoporous graphene. Science 360, 199-203 (2018).

19. Elias, D. C. et al. Control of graphene's properties by reversible hydrogenation: evidence for graphane. Science 323, 610-613 (2009).

20. Balog, R. et al. Bandgap opening in graphene induced by patterned hydrogen adsorption. Nat. Mater. 9, 315-319 (2010).

21. Jørgensen, J. H. et al. Symmetry-driven band gap engineering in hydrogen functionalized graphene. ACS Nano 10, 10798-10807 (2016).

22. Li, H. et al. Site-selective local fluorination of graphene induced by focused ion beam irradiation. Sci. Rep. 6, 19719 (2016).

23. Altenburg, S. J., Lattelais, M., Wang, B., Bocquet, M. L. \& Berndt, R. Reaction of phthalocyanines with graphene on $\operatorname{Ir(111).~J.~Am.~Chem.~Soc.~137,~9452-9458~(2015).~}$

24. Greenwood, J. et al. Covalent modification of graphene and graphite using diazonium chemistry: tunable grafting and nanomanipulation. ACS Nano 9, 5520-5535 (2015).

25. He, Y. Q. et al. Fusing tetrapyrroles to graphene edges by surface-assisted covalent coupling. Nat. Chem. 9, 33-38 (2017).

26. Daukiya, L. et al. Covalent functionalization by cycloaddition reactions of pristine defect-free graphene. ACS Nano 11, 627-634 (2017). 
27. Criado, A., Melchionna, M., Marchesan, S. \& Prato, M. The covalent functionalization of graphene on substrates. Angew. Chem. Int. Ed. 54, 10734-10750 (2015).

28. Bian, S. et al. Covalently patterned graphene surfaces by a force-accelerated Diels-Alder reaction. J. Am. Chem. Soc. 135, 9240-9243 (2013).

29. Cao, Y., Osuna, S., Liang, Y., Haddon, R. C. \& Houk, K. N. Diels-Alder reactions of graphene: computational predictions of products and sites of reaction. J. Am. Chem. Soc. 135, 17643-17649 (2013).

30. Navarro, J. J. et al. Organic covalent patterning of nanostructured graphene with selectivity at the atomic level, Nano Lett. 16, 355-361 (2016).

31. Navarro, J. J., Calleja, F., Miranda, R. E., Pérez, M. \& Vázquez de Parga, A. L. High yielding and extremely site-selective covalent functionalization of graphene, Chem. Commun. 53, 1041810421 (2017).

32. Liu, L. H., Lerner, M. M. \& Yan, M. Derivitization of pristine graphene with well-defined chemical functionalities. Nano Lett. 10, 3754-3756 (2010).

33. Denis, P. A. \& Iribarne, F., Cooperative behavior in functionalized graphene: explaining the occurrence of 1,3 cycloaddition of azomethine ylides onto graphene. Chem. Phys. Lett. 550, 111117 (2012).

34. MacLeod, J. M. \& Rosei, F. Molecular self-assembly on graphene. Small 10, 1038-1049 (2014).

35. Kelly, R.E.A. \& Kantorovich, L. Planar nucleic acid base super-structures. J. Mat. Chem. 16, 19841905 (2006).

36. Wang, Q. H. et al. Understanding and controlling the substrate effect on graphene electrontransfer chemistry via reactivity imprint lithography. Nat. Chem. 4, 724-732 (2012).

37. Khomyakov, P. A. et al. First-principles study of the interaction and charge transfer between graphene and metals. Phys. Rev. B 79, 195425-195436 (2009).

Acknowledgements: This work was financially supported by the National Natural Science Foundation of China (21473045, 51772066, 21603086), the Engineering and Physical Sciences Research Council (EP/L000202, EP/P020194), and State Key Laboratory of Urban Water Resource and Environment (2018DX04). F.R. acknowledges partial salary support from the Canada Research Chairs program.

Author contributions: A.G., M. Y. and F. B. designed the project; C. M. and A. G. synthesized the BCM molecule; C. C., G. S., S. W. and Z. L. conducted the graphene growth/BCM-graphene reaction/STM imaging/Raman analysis; W. H., C. C. and M. C. collected the IRAS results; K. S., F. S., Z. L. and J. P. 
carried out the ARPES studies; Q. L., L. K., H.S., P. D. and P. G. performed the calculations; M. Y., C. C., Y. S., M. C., F. R., L. K. and F. S. analyzed and interpreted the results; M. Y., C. C., L. K. and F. R. wrote the manuscript; F. B. advised the project process.

Competing interests: The authors declare no competing interests.

\section{Figure legends}

Fig. 1| Schematic illustration for the photocycloaddition of BCM layer with graphene on $\mathrm{Cu}(111)$.

BCM compound is maleimide derivative equipped with 3,5-bis(carboxylic acid)-phenyl ring, where the carbon, oxygen, nitrogen, and hydrogen atoms are in light grey, red, blue, and white, respectively. Its $\mathrm{C}=\mathrm{C}$ of the maleimide group is designed for the cycloaddition reaction with graphene layer, as highlighted in the white ellipse; its carboxylic acid groups in the meta-position are set for the formation of extended molecular network by intermolecular hydrogen bonding as well as for enhancing the reactivity of the maleimide. As depicted by the three-dimensional STM image (in blue), upon ultraviolet irradiation in ultrahigh vacuum, the photocycloaddition between the BCM layer and single-layer graphene is triggered, with the reaction sites being spatially selective and well ordered.

Fig. 2 | Self-assembled BCM network on the graphene-Cu(111) substrate. $a$, Large-scale and $b$, highresolution STM images of the single-layer graphene epitaxially grown on $\mathrm{Cu}(111)$ with ethyne as precursor, resulting in a highly crystalline full layer. $\mathbf{c}$ and $\mathbf{d}$, The long-range ordered BCM network on the single-layer graphene-Cu(111), which are tiled by building blocks of hexapetalous flowers each consisting of six BCM molecules. The flower-like units are marked in different colors in panel d. e, DFT optimized three-dimensional (3D) model of BCM on graphene-Cu(111), showing that all the molecules are parallel to the substrate in a flat conformation. $\mathbf{f}$, Calculated model of a single building block of the network, revealing that the hexapetalous flower-like motif is stabilized by intermolecular double $\mathrm{O}-\mathrm{H} \cdots \mathrm{O}$ hydrogen bonds between the neighboring carboxylic acid groups. g, DFT optimized BCM network on the graphene layer (the Cu substrate is not shown for clarity), where the unit cell is $10.9^{\circ}$ deviated from the graphene symmetry direction as highlighted by the white rhombus. $\mathbf{h}$ and $\mathbf{i}$, Zoom-in experimental and simulated STM images of the BCM network on graphene-Cu(111), which fit well with each other, presenting an identical tunneling contrast for all molecules.

Fig. 3 Cycloaddition of the BCM network on graphene-Cu(111). a, Large-scale and b, highresolution STM images of the BCM network on graphene- $\mathrm{Cu}(111)$ after UV irradiation. While the arrangement of the network remains the same, the tunneling contrast and morphology of four 
molecules in each unit cell have been modified by the treatment. The varied four molecules are denoted as ' $\alpha$ ', and the other two are marked as ' $\beta$ '. c and d, DFT optimized 3D and 2D models of the cycloaddition between the molecular layer and graphene- $\mathrm{Cu}(111)$, indicating that cycloaddition is spatially selective and only occurs for the molecules at specific positions relative to the graphene layer. In panel $\mathrm{d}$, the unreacted $\beta$ molecules are in golden brown, and the copper slab is excluded for clarity. e, Zoom-in model for the six BCM in each unit (marked in panel d), indicating that only the four $\alpha$ molecules have an appropriate angle and distance for covalent bonding with the underlying graphene whilst $\beta$ cannot enable the bonding, where $\alpha 1$ and $\bar{\alpha} 1$ are in $C 4$, and $\alpha 2$ and $\bar{\alpha} 2$ are in $C 2$ cycloaddition, respectively. $f$, Simulated STM image, which is in good agreement with the experimental observation and indicates that the reacted molecules have a lower contrast than the unreacted ones.

Fig. 4| Vibrations and band structure of the BCM network on graphene- $\mathrm{Cu(111).} \mathrm{a,} \mathrm{Infrared}$ reflection-absorption spectra of various samples, where 'UV1'/'UV2' represent the cases of BCM on graphene after the first/second-round UV irradiation. b, Modeling of a single BCM in $C 2[2+2] / C 4[2+4]$ cycloaddition with graphene and the new vibrations due to the reaction. $c$, Band mapping along the $\bar{\Gamma}-\overline{\mathrm{K}}$ direction and $\mathbf{d}$, the close view at the $\mathrm{K}$ point of the pristine graphene on $\mathrm{Cu}(111)$, showing the linear dispersion of the typical Dirac band. e, Band structure around the Dirac point for the reacted graphene, where the gap opening (marked by the thin blue lines) is evident.

\section{Methods}

Synthesis of single-layer graphene on $\mathrm{Cu}(\mathbf{1 1 1})$ : Highly crystalline single-layer graphene was grown epitaxially on the $\mathrm{Cu}(111)$ substrate under ultrahigh vacuum (UHV) conditions at a base pressure of $3.0 \times 10^{-10} \mathrm{mbar}$, by chemical vapor deposition. Ethyne was used as precursor, and its pressure was controlled by a leak valve mounted on the UHV chamber. The clean $\mathrm{Cu}(111)$ substrate was heated to $993 \mathrm{~K}$, and the ethyne pressure was sequentially increased to $6.0 \times 10^{-6} \mathrm{mbar}$ and maintained for 20 $\min$. The surface was then annealed for another 20 min with the gas valve off. Using this method, a full-coverage, high-quality graphene layer can be obtained with only one growth cycle.

Experimental details: The single-crystal $\mathrm{Cu}(111)$ substrate was cleaned by repeated cycles of Argon ion sputtering (1.0 KeV) and annealing (800 K). 3,5-bis(carboxylic acid)-phenyl-3-maleimide (BCM) compound was thermally sublimated ( $420 \mathrm{~K})$ from a molecular evaporator and deposited onto the graphene-Cu(111) substrate kept at room temperature (RT). No post-annealing was applied for the formation of the BCM network. Ultraviolet (UV) irradiation was carried out using a UV lamp (GGZ500, 
Shanghai Jiguang Lighting Factory), with a central wavelength of $365 \mathrm{~nm}$. Based on the experimental settings, the irradiation power density was measured using a light intensity detector (mL-01 SiPyranometer, Japan) at the applied sample-lamp distance $(\sim 20 \mathrm{~cm})$ with the glass viewport in between, deducing a power density of $40 \mathrm{~mW} \cdot \mathrm{cm}^{-2}$ on the sample surface. The UV lamp was not focused so that the entire sample was irradiated. Both scanning tunneling microscopy (STM) and infrared reflection-absorption spectroscopy (IRAS) studies were carried out on the epitaxially-grown graphene-Cu(111) substrate in UHV, following an identical procedure of sample preparation. All STM images were scanned in the constant current mode at RT, using a variable-temperature Aarhus STM ${ }^{38}$ with a negative bias voltage of $1.0-1.3 \mathrm{~V}$ on the sample and a tunneling current of $0.2-0.5 \mathrm{nA}$. The STM scanning area was up to $1000 \AA \times 1000 \AA$ in size. To acquire sufficient data for statistical analysis on the coverage of the molecular layer, more than 20 different randomly-selected scanning areas were probed after each sample treatment step. For the UV irradiation treatment, the sample was exposed to the UV light first, then transferred to the STM stage in the same UHV chamber for scanning. The IRAS measurements were carried out using an infrared spectrometer (Bruker VERTEX 70V, U.S. A.) with ZnSe window and mercury cadmium telluride detector. The size of the optical source was $\sim 3 \mathrm{~mm}$. Given the small grazing incidence angle used for IRAS, the detected zone was up to $\sim 1.0 \mathrm{~cm}$ in size. Raman analysis was carried out on a free-standing graphene (purchased from Changzhou Carbon Time Technology Co., Ltd., China.) using an inVia Raman spectrometer (Renishaw, U.K.) with incident wavelength of $532 \mathrm{~nm}$. Angle resolved photoemission spectroscopy (ARPES) measurements ${ }^{39}$ were carried out at the Dreamline (BLO9U) in Shanghai Synchrotron Radiation Facility using a VG-Scienta DA30 electron analyser with a base pressure of $\sim 5 \times 10^{-11} \mathrm{mbar}$ and the sample kept at $5 \mathrm{~K}$. The photon energy was set at $70 \mathrm{eV}$ and calibrated by measuring the Fermi level of the metal sample plate. The angular resolution was $0.2^{\circ}$; the energy resolution was $5 \mathrm{meV}$.

Calculation methods: All our relaxation calculations were based on the density functional theory (DFT) as implemented in the VASP package ${ }^{40}$ using plane waves as a basis set with $550 \mathrm{eV}$ plane-wave cutoff, norm-conserving pseudo-potentials and the Perdew-Burke-Ernzerhof (PBE) exchangecorrelation density functional ${ }^{41}$. The van der Waals interaction was taken into account within the Grimme D3 approach ${ }^{42}$. The force convergence criterion used for the geometry optimization was $0.005 \mathrm{eV} / \AA$. . The excitation spectra were calculated using linear-response time-dependent DFT (TDDFT) calculations as implemented in the CP2K Quickstep package ${ }^{43,44}$ employing a hybrid Gaussian and plane-wave method. It calculates the ground state first, and subsequently the time-dependent electron response to an external field to obtain excitation energies, and their corresponding dipole oscillator strengths. The $\mathrm{Cu}(111)$ surface was constructed using three atomic $\mathrm{Cu}$ layers, where the bottom two layers were frozen during geometry optimization, with a vacuum gap (the distance 
between the molecular network and the bottom surface of the next slab) of $25 \AA \AA$. In the calculations of excitation states, a single BCM molecule and a graphene substrate of 200 atoms were considered. Climbing Image Nudged Elastic Band (CI-NEB) ${ }^{45,46}$ calculations were performed as implemented in the VASP code to find the barrier between the covalently-bonded (reacted) and physisorbed (unreacted) states of a single molecule on the substrate.

\section{References}

38. Laegsgaard, E. et al. A high-pressure scanning tunneling microscope. Rev. Sci. Instrum. 72, 35373542 (2001).

39. Shen, K. et al. Fabricating quasi-free-standing graphene on a $\mathrm{SiC}(0001)$ surface by steerable intercalation of iron, J. Phys. Chem. C, 122, 21484-21492 (2018).

40. Kresse, G. et al. Efficient iterative schemes for ab initio total-energy calculations using a planewave basis set. Phys. Rev. B 54, 11169-11186 (1996).

41. Perdew, J. P. et al. Generalized gradient approximation made simple. Phys. Rev. Lett. 77, 38653868 (1996).

42. Grimme, S. et al. A consistent and accurate $a b$ initio parametrization of density functional dispersion correction (DFT-D) for the 94 elements H-Pu. J. Chem. Phys. 132, 154104 (2010).

43. Vande Vondele, J. et al. Quickstep: Fast and accurate density functional calculations using a mixed Gaussian and plane waves approach. Comput. Phys. Commun. 167, 103-128 (2005).

44. Hutter, J. et al. CP2K: atomistic simulations of condensed matter systems. WIREs Comput. Mol. Sci. 4, 15-25 (2014).

45. Jónsson, H., Mills, G. \& Jacobsen, K. W. Nudged elastic band method for finding minimum energy paths of transitions, in classical and quantum dynamics in condensed phase simulations, Ed. B. J. Berne, G. Ciccotti and D. F. Coker, 385 (World Scientific, 1998).

46. Henkelman, G. \& Jonson, H. A climbing image nudged elastic band method finding saddle points and minimum energy paths, J. Chem. Phys. 113, 9901-9904 (2000).

Data availability: The methods and materials used in this study are available in the Supplementary Information. The authors declare that all data supporting the findings of this study are available 
within the paper [and its supplementary information files]. Correspondence and requests for materials should be addressed to M.Y, A.G., L.K, or F. B. 


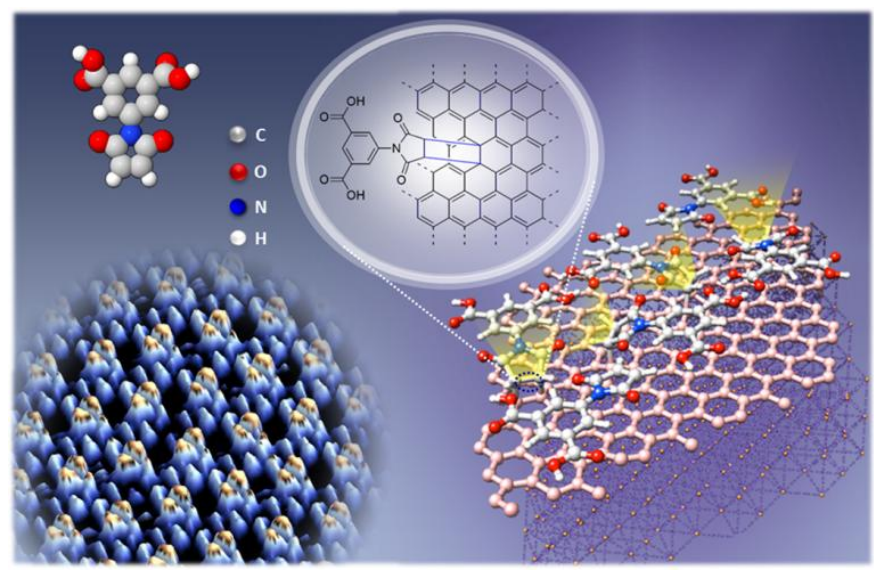



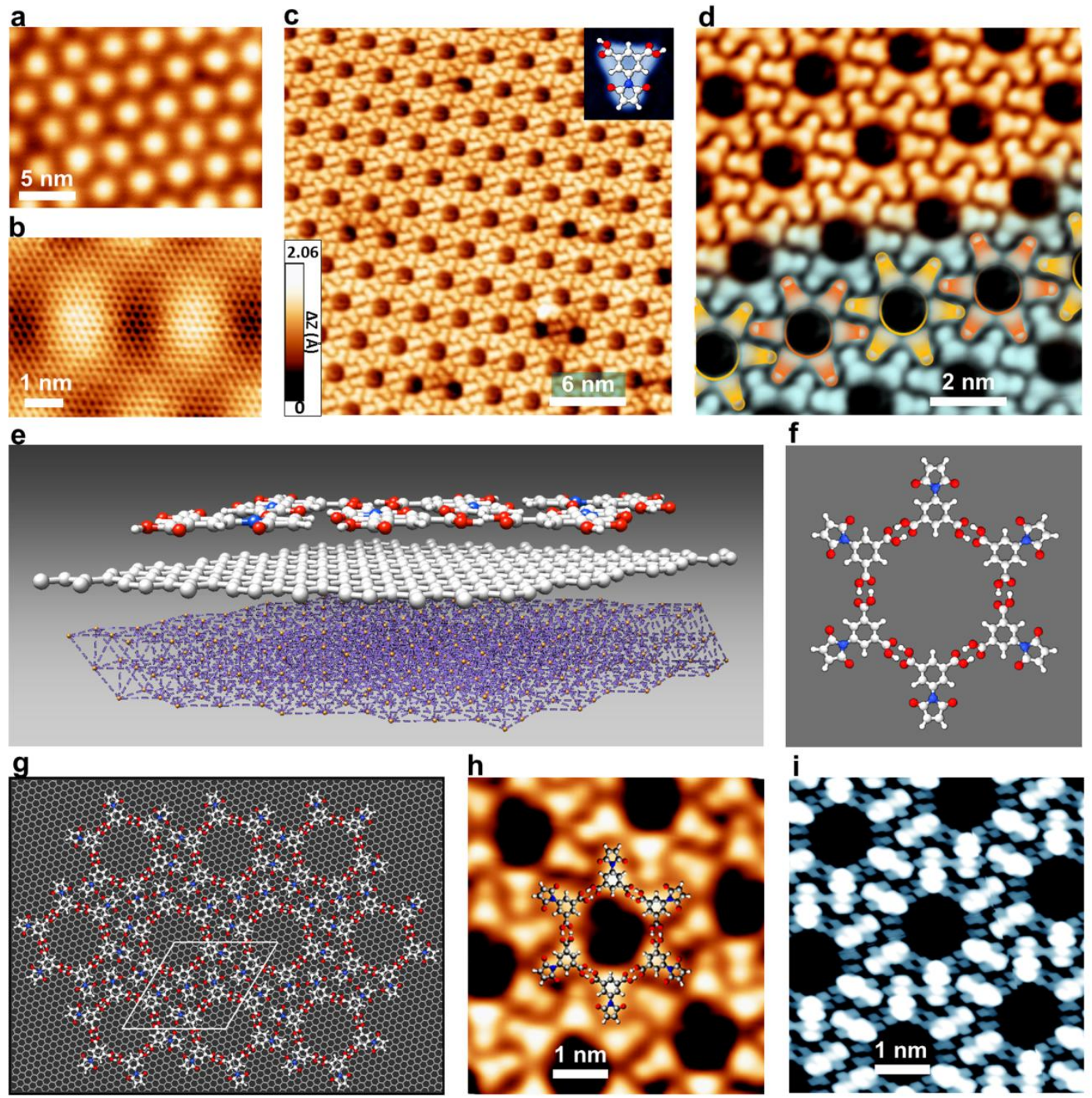

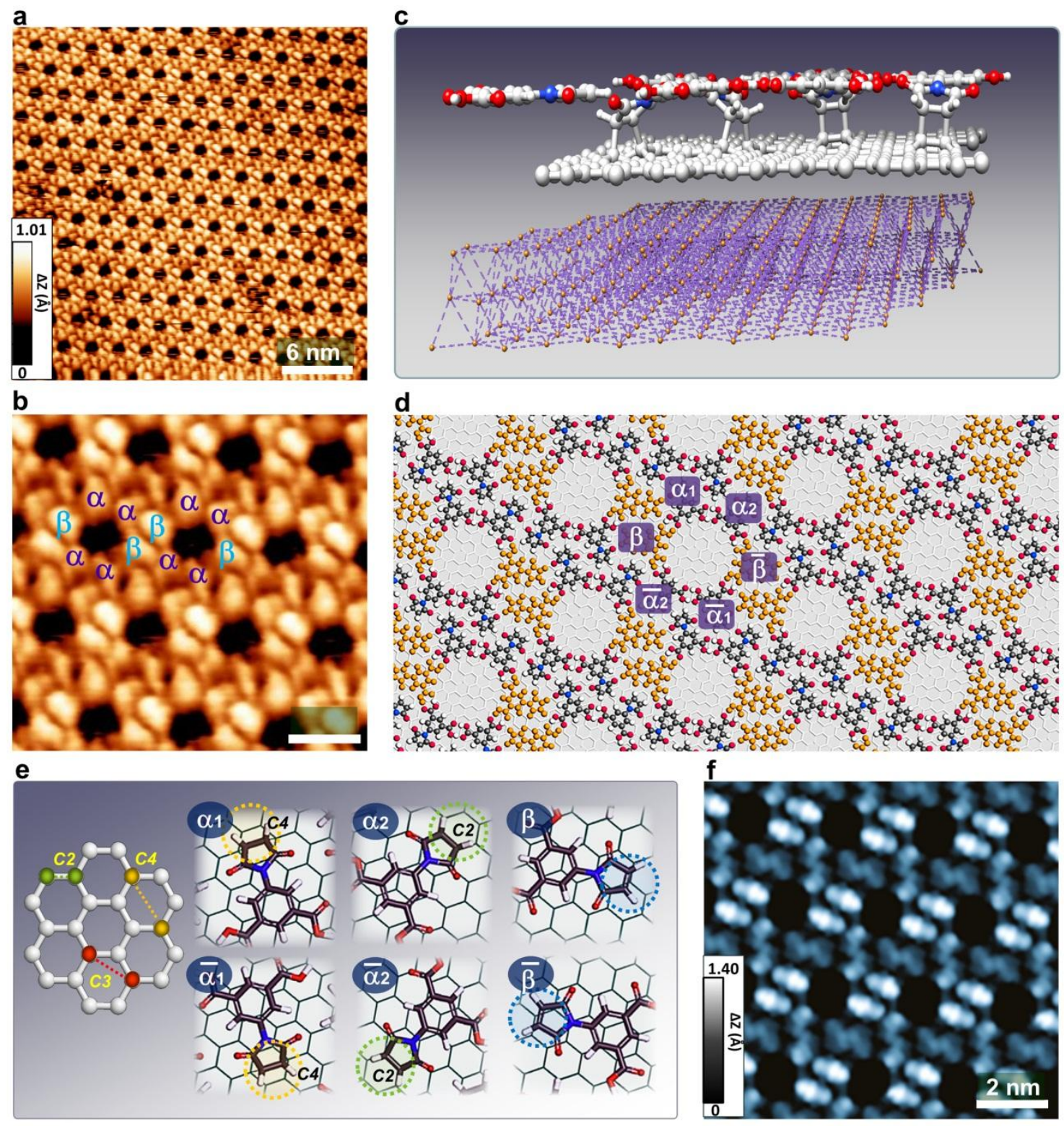

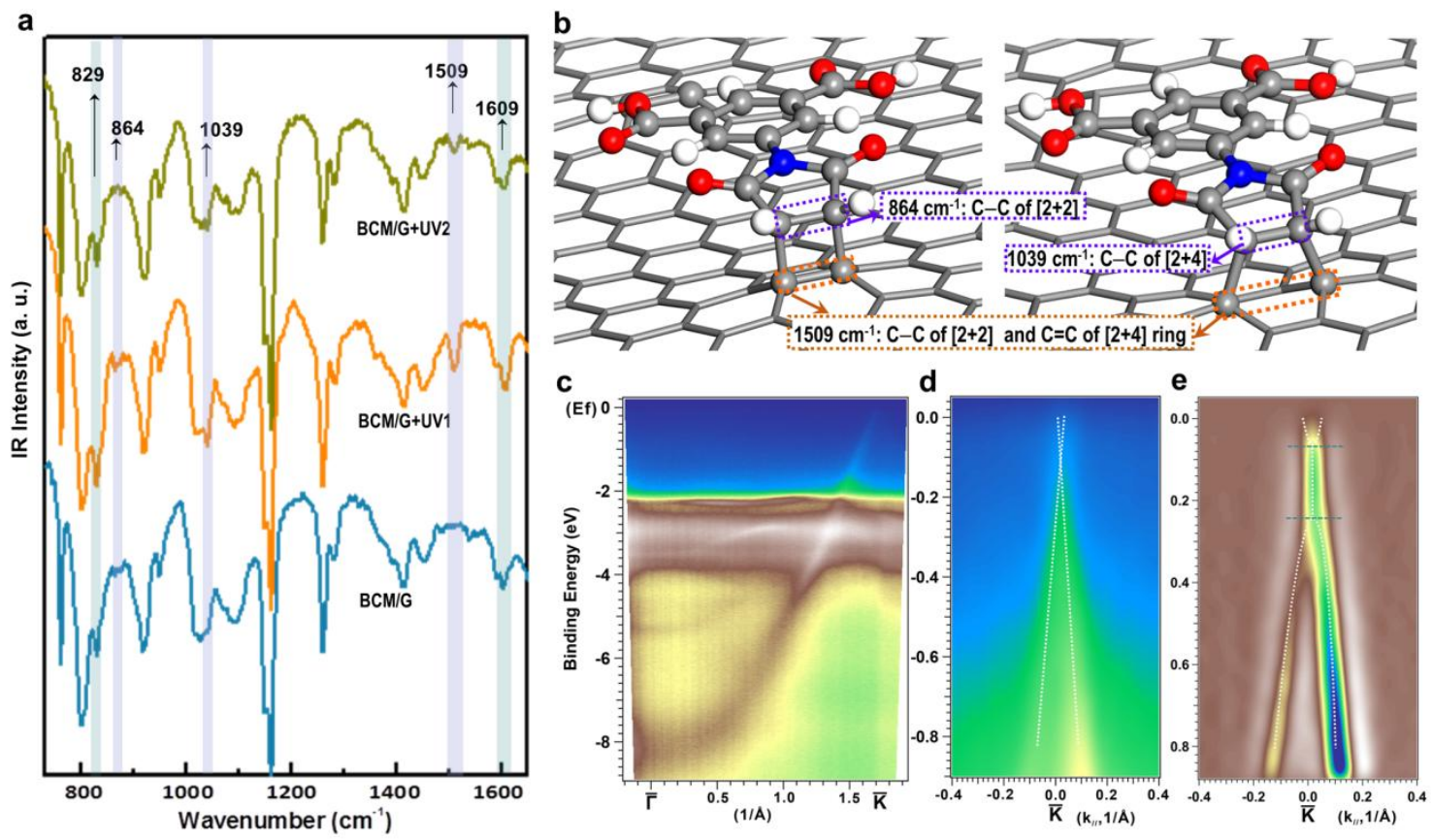


\section{Long-range ordered and atomic-scale control of graphene hybridization by photocycloaddition}

Miao $\mathrm{Yu}^{1, \#, *}$, Chong Chen ${ }^{1, \#}$, Qi Liu ${ }^{3, \#}$, Cristina Mattioli ${ }^{2}$, Hongqian Sang ${ }^{4,5}$, Guoqiang Shi ${ }^{1}$, Wujun Huang ${ }^{6}$, Kongchao Shen ${ }^{7}$, Zhuo $\mathrm{Li}^{1}$, Pengcheng Ding ${ }^{8}$, Pengfei Guan ${ }^{3}$, Shaoshan Wang ${ }^{1}$, Ye Sun ${ }^{8}$, Jinping $\mathrm{Hu}^{7}$, André Gourdon ${ }^{2, *}$, Lev Kantorovich ${ }^{4, *}$, Flemming Besenbacher ${ }^{9, *}$, Mingshu Chen ${ }^{6}$, Fei Song ${ }^{7}$, and Federico Rosei ${ }^{10}$

1. State Key Laboratory of Urban Water Resource and Environment, School of Chemistry and Chemical Engineering, Harbin Institute of Technology, Harbin 150001, China

2. CEMES-CNRS, Toulouse 31055, France

3. Beijing Computational Science Research Center, Beijing 100193, China

4. Department of Physics, King's College London, The Strand, London WC2R 2LS, U.K.

5. Institute for Interdisciplinary Research, Jianghan University, Wuhan 430056, China

6. Department of Chemistry, Xiamen University, Xiamen 361005, China

7. Shanghai Synchrotron Radiation Facility, Shanghai Advanced Research Institute, Chinese Academy of Sciences, Shanghai 201200, China

8. Condensed Matter Science and Technology Institute, Harbin Institute of Technology, Harbin 150001, China

9. iNANO and Department of Physics and Astronomy, Aarhus University, Aarhus 8000, Denmark 10. INRS Centre for Energy, Materials and Telecommunications, Varennes J3X 1S2, Canada

\# These authors contributed equally to this work. 
Correspondence to: miaoyu_che@hit.edu.cn, andre.gourdon@cemes.fr, lev.kantorovitch@kcl.ac.uk, and fbe@inano.au.dk

1. Long-range ordered, full coverage of BCM molecular layer on graphene-Cu(111)
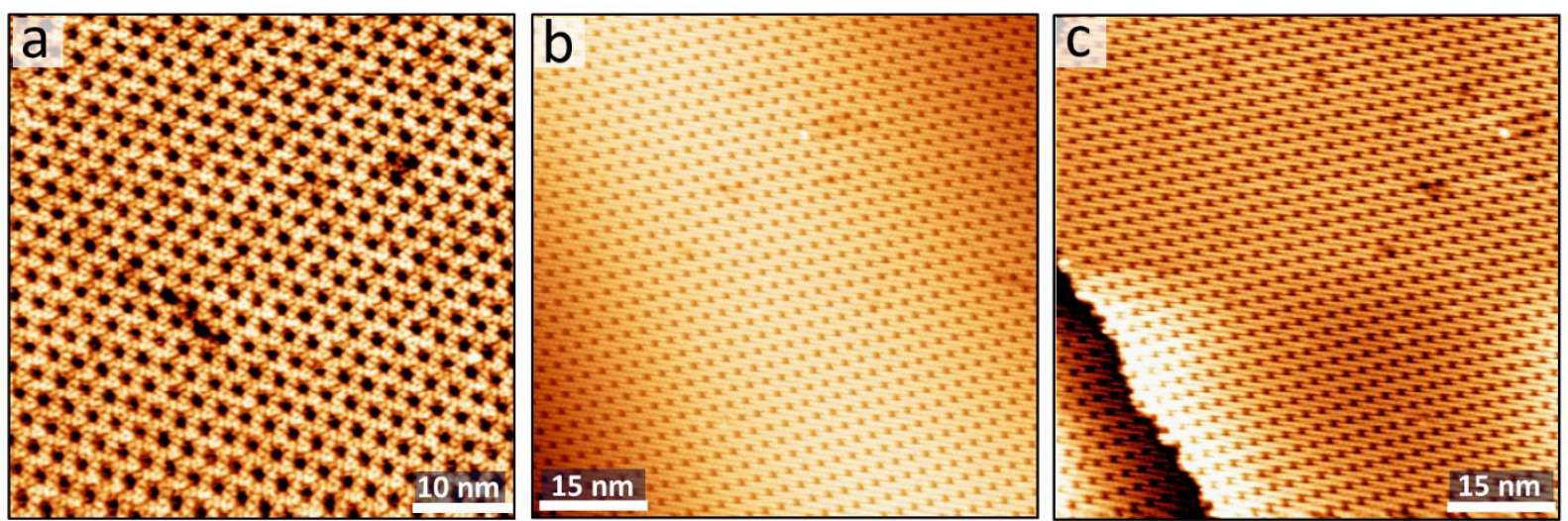

Supplementary Fig. 1| STM images of the self-assembled monolayer of BCM molecules on graphene-Cu(111), covering the entire surface including the flat terraces and step edges. 


\section{Electron transfer between the BCM and graphene layer}

Before irradiation, all molecules in the $\mathrm{BCM}$ layer lie on the graphene- $\mathrm{Cu}(111)$ substrate in a flat geometry. The calculated electron density difference is shown in Supplementary Figs. 2a-b, revealing no significant electron transfer between the $\mathbf{B C M}$ and graphene layer. The results indicate a typical physisorption of the $\mathrm{BCM}$ on graphene-Cu(111). In sharp contrast, an evident covalent bonding is established between four BCM molecules in each unit cell and the graphene sheet after UV irradiation (Supplementary Figs. 2c-e).
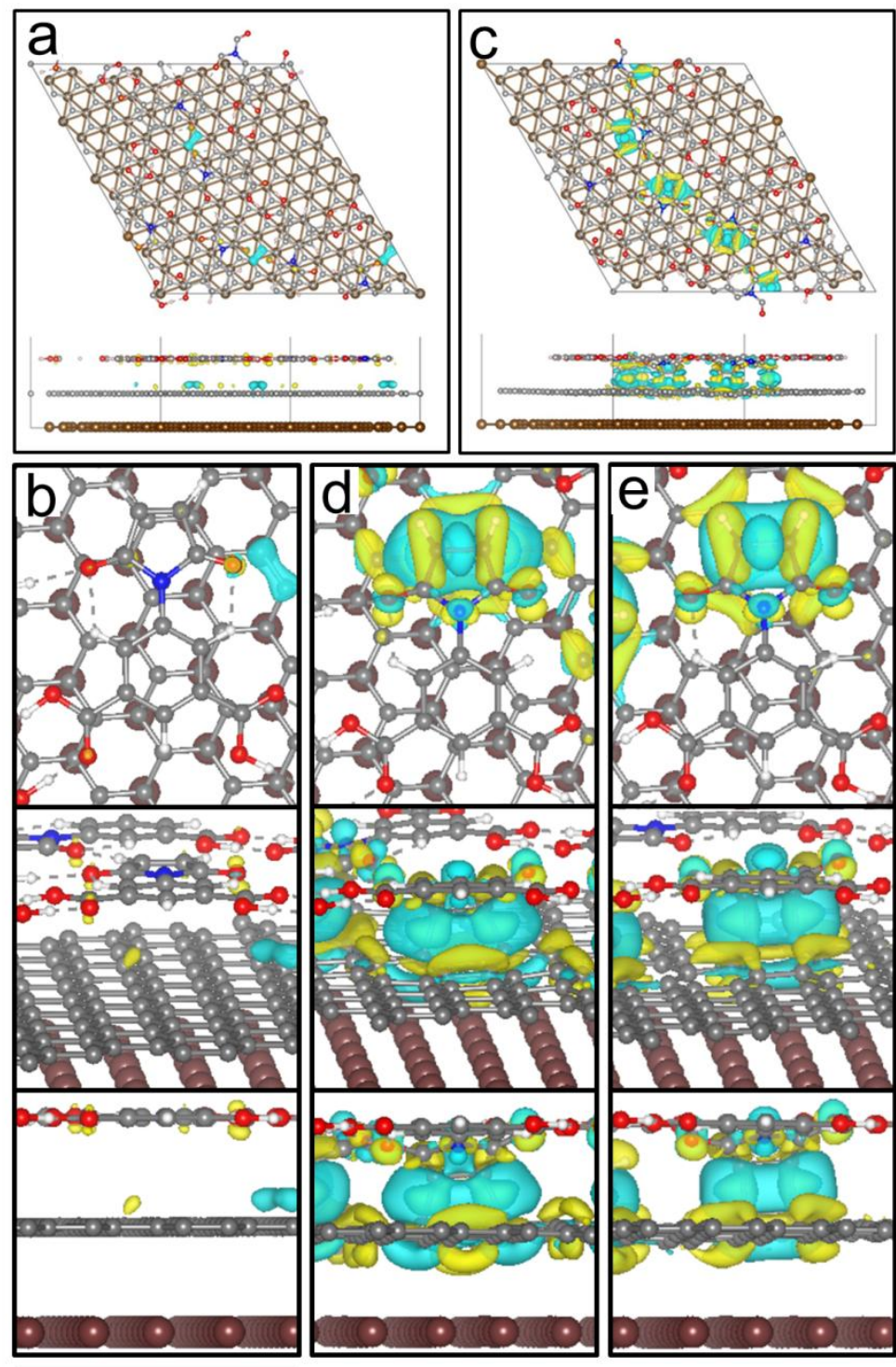

Supplementary Fig. 2| The electron density difference (EDD) iso-surface maps between the molecular network and the graphene on $\mathrm{Cu}(111)$ surface. a, Top and side views of BCM network before irradiation (the isovalue is 0.0005 a.u.); $\mathbf{b}$, Topview, tilted, side-view model of a BCM in the network before irradiation (the isovalue is 0.0005 a.u.); c, Top and side views 
of BCM network after irradiation (the isovalue is 0.005 a.u.); Top-view, tilted, side-view model of a BCM in d, C4 cycloaddition and e, $C 2$ cycloaddition in the network after irradiation (the isovalue is 0.002 a.u.). Only the outmost $\mathrm{Cu}$ layer is shown for simplicity.

\section{Charge transfer of a single BCM within the molecule layer before irradiation}

The building block of the BCM network on graphene- $\mathrm{Cu}(111)$ is a hexapetalous-flower unit. Within the unit, the six $\mathrm{BCM}$ are associated with intermolecular double $\mathrm{O}-\mathrm{H} \cdots \mathrm{O}$ hydrogen bonds between the adjacent carboxylic acid groups of the neighboring BCM. The units are then tiled into an extended network. The charge distribution related to the hydrogen bonds of each BCM can be revealed in the charge density difference plot that is presented in Supplementary Fig. 3 , where the red and blue colors represent depletion and excess electronic regions, respectively.

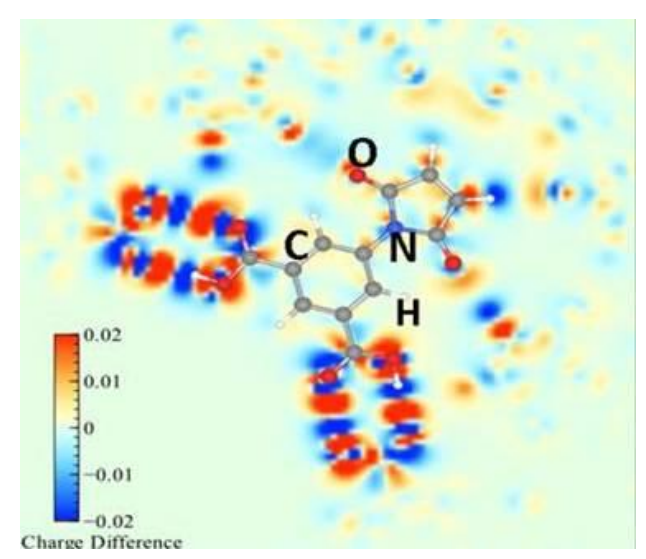

Supplementary Fig. 3| The charge distribution related to the hydrogen bonds of each BCM (i.e., the charge density difference). 
4. The orientation of the $\mathrm{BCM}$ network relative to the graphene layer

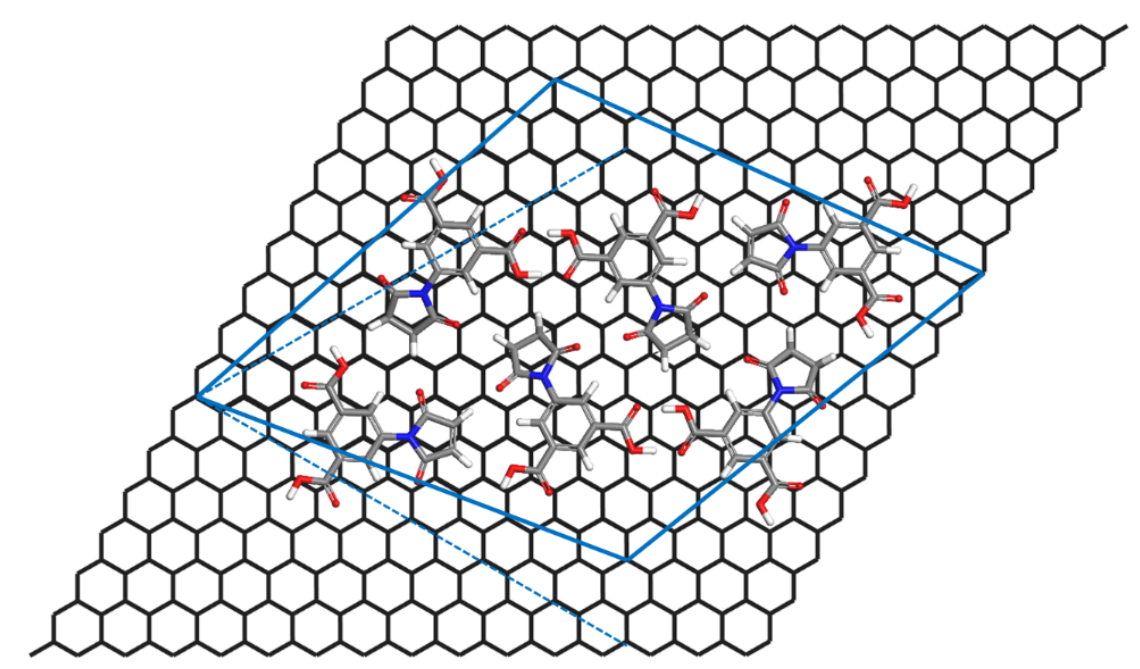

Supplementary Fig. 4 | The orientation of the BCM network relative to the underlying graphene, showing a rotation of $10.9^{\circ}$. 


\section{Large-scale STM images of BCM adlayer on graphene-Cu(111) after irradiation}
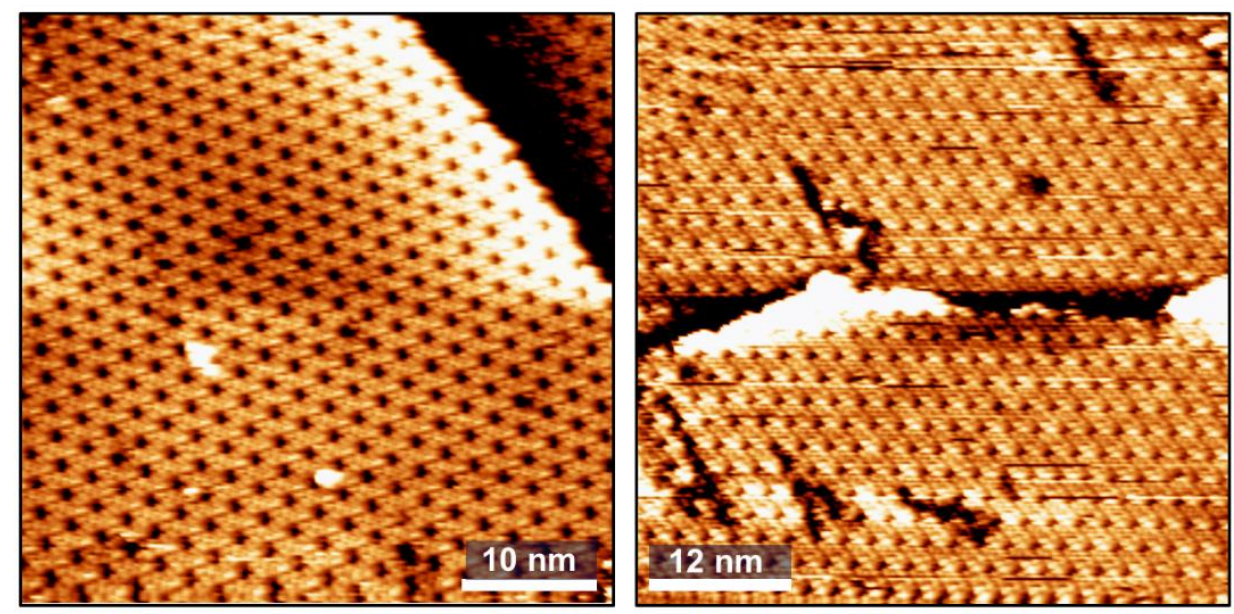

Supplementary Fig. 5| STM images of BCM adlayer on graphene-Cu(111) after UV irradiation.

\section{Energies of the BCM layer on graphene-Cu(111)}

The total energies calculated for various systems are summarized in Supplementary Table 1. Based on the results, the energy of each unit of the $\mathbf{B C M}$ molecular layer $(\mathrm{ML})$ on graphene-Cu(111) is increased (becomes less negative) by $8.26 \mathrm{eV}\left(E_{6 M+S}^{\text {non-flat }}-E_{6 M+S}^{\text {flat }}=8.26 \mathrm{eV}\right)$ upon the cycloaddition. Neglecting the small contribution from the two unreacted molecules, the average energy increase for each BCM cycloaddition is around $2.06 \mathrm{eV}$. To enable covalent bonding, both BCM and graphene at the reaction site have been seriously deformed. The deformation energy of $\mathbf{B C M}$ and graphene contributes dominantly to the overall energy increase; besides, it is related to the hybridization varied from $s p^{2}$ to $s p^{3}$, the non-optimized bonds between BCM and graphene, and the modified intermolecular interaction with the BCM layer. Consistently, earlier theoretical studies also predicted that a cycloaddition for a single molecule with the bulk of defect-free graphene layer could cost up to $2.6 \mathrm{eV}^{1,2}$.

The interaction energies of the flat and non-flat ML (as a whole) with the graphene- $\mathrm{Cu}(111)$ substrate are $E_{\text {lnt }}^{\text {flat }}=E_{6 M+S}^{\text {flat }}-E_{6 M}^{\text {flat }}-E_{S}^{\text {flat }}=-6.99 \mathrm{eV}$, and $E_{\text {lnt }}^{\text {non-flat }}=E_{6 M+S}^{\text {non-flat }}-E_{6 M}^{\text {non-flat }}-E_{S}^{\text {non-flat }}=-15.33 \mathrm{eV}$. Assuming that the six BCM in the flat geometry are equivalent, the interaction energy per molecule for the flat $\mathrm{ML}$ is $-6.99 / 6=-1.17 \mathrm{eV}$. Also assuming that the interaction energy of each unreacted molecule in the non-flat ML does not significantly change from that before irradiation, the average interaction energy of the four reacted molecules with the graphene- $\mathrm{Cu}(111)$ substrate is, therefore, $(-15.33-2 \times(-1.17)) / 4=-3.25 \mathrm{eV} /$ molecule, which is by nearly $2.09 \mathrm{eV}$ more stable than that before reaction.

Supplementary Table 1. Energies used in our analysis of the energetics for binding of the ML to the substrate (S). 


\begin{tabular}{|c|c|c|}
\hline The system & Notation & VASP energy (eV) \\
\hline $\mathrm{ML}$ (six molecules (M)) on S, in the flat geometry & $E_{6 M+S}^{\text {flat }}$ & -4502.18 \\
\hline $\mathrm{ML}(\operatorname{six} \mathrm{M}$ ) on $\mathrm{S}$, in the non-flat geometry & $E_{6 M+S}^{\text {non-flat }}$ & -4493.92 \\
\hline $\mathrm{ML}($ six $\mathrm{M})$, in the flat geometry & $E_{6 M}^{\text {flat }}$ & -1106.55 \\
\hline $\mathrm{ML}($ six $\mathrm{M})$, in the non-flat geometry & $E_{6 M}^{\text {non-flat }}$ & -1099.36 \\
\hline Graphene on $\mathrm{Cu}(111)$, in the flat geometry & $E_{s}^{\text {flat }}$ & -3388.64 \\
\hline Graphene on $\mathrm{Cu}(111)$, in the non-flat geometry & $E_{s}^{\text {non-flat }}$ & -3379.23 \\
\hline Single $\mathrm{M}$, gas phase & $E_{M}$ & -183.42 \\
\hline
\end{tabular}

*Note that the flat geometry corresponds to the state of the system before reaction, and the non-flat geometry is attributed to the one after reaction. ML corresponds to six molecules in the unit cell and the substrate includes a graphene layer on top of a $\mathrm{Cu}(111)$ slab. All energies include the van der Waals interaction.

\section{IRAS results of the BCM network on graphene-Cu(111) upon UV irradiation}

The full and zoom-in IRAS results of BCM on graphene- $\mathrm{Cu}(111)$ are presented in Fig.4a and Supplementary Fig. 6. To assign the vibration peaks, DFT and density functional perturbation theory (DFPT) were employed. First, the models of a single BCM on a single-layer graphene in (a) physisorption, (b) C2 ([2+2]) cycloaddition, and (c) C4 ([2+4]) were optimized with DFT (Supplementary Fig. 7), until the maximum force on each atom was smaller than $0.01 \mathrm{eV} / \AA ̊$. The normal-mode vibrations of these three configurations were then calculated using DFPT. Both DFT and DFPT calculations were carried out using VASP code (with plane wave cut-off energy of $550 \mathrm{eV}$ ) and the PAW-PBE pseudopotentials.

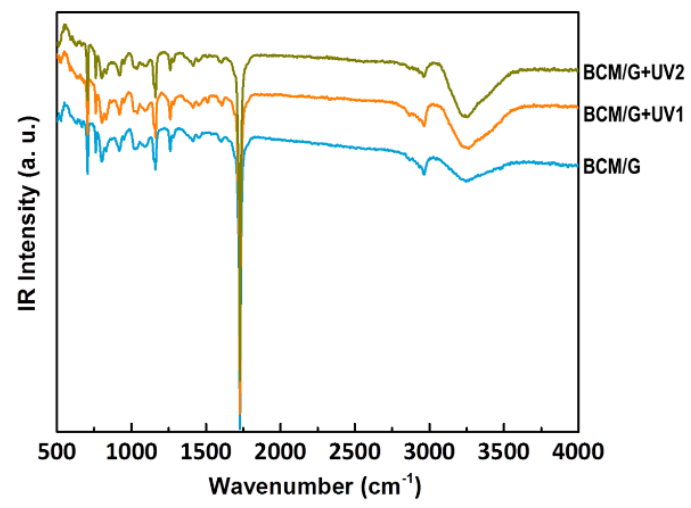

Supplementary Fig. 6| Full infrared reflection-absorption spectra of BCM on graphene-Cu(111). 

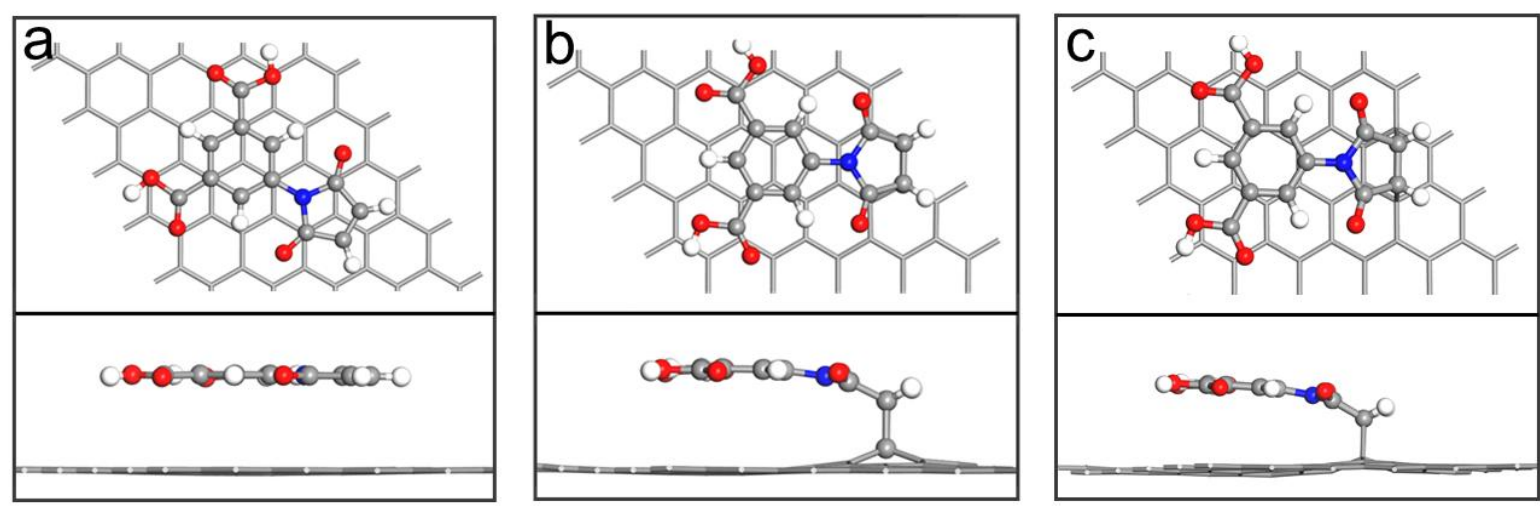

Supplementary Fig. 7| Top- and side-view of a single BCM in a, physisorption, b, $C 2([2+2])$ and c, $C 4([2+4])$ cycloaddition with a single-layer graphene.

\section{Raman results for the $\mathrm{BCM}$ layer on quasi-free-standing graphene}

Raman analysis (Supplementary Fig. 8) was carried out on a quasi-free-standing graphene on mica. Before depositing the BCM molecule, the pristine graphene was pre-treated by annealing in UHV ( $\sim 673 \mathrm{~K}$ for $60 \mathrm{~min}$ ) to remove possible contaminants. The molecule deposition and UV irradiation were carried out in UHV, same as the sample preparation for STM/IRAS. The samples were then removed from the UHV chamber and analyzed using Raman spectroscopy under ambient conditions. The focused optical source had a size of $\sim 2 \mu \mathrm{m}$. For each preparation step, the representative spectra were measured at 15 randomly selected areas of the sample. Raman spectrum of the ' $B C M / G+U V 1$ ' was collected from the sample of BCM network on the quasi-free-standing graphene after 8-min UV irradiation in UHV. In the case of ' $B C M / G+U V 2$ ', the sample was irradiated for 8 min for the first round; and $1 \mathrm{~h}$ later, it was irradiated for another $11 \mathrm{~min}$ as the second round without taking it out from the UHV chamber. Since all samples were prepared and treated in UHV under the same conditions and just measured under ambient conditions, the reaction can be guaranteed to proceed in the same way. No noticeable disturbance from impurities was observed in the Raman results.

The Raman spectrum of the pristine graphene sample presents strong peaks associated with the G-band $\left(1585.6 \mathrm{~cm}^{-1}\right)$ and 2D-band $\left(2686.1 \mathrm{~cm}^{-1}\right)$, while the D-band corresponding to the defects of the graphene layer is rather weak with a relative intensity of the $D$-band to the $G$-band $\left(I_{D} / I_{G}\right)$ of 0.068. Depositing the BCM layer and prior to UV irradiation, the Raman spectrum shows almost no variation from the case of pristine graphene. In contrast, after irradiation, the relative intensity of the D-band evidently enhances $\left(I_{D} / I_{G}\right.$ of 0.17$)$. The reversibility of the reaction is also confirmed by the Raman results. After the second round of UV irradiation for $11 \mathrm{~min}$, the relative intensity of the Dband of Raman spectrum decreases $\left(I_{D} / I_{G}\right.$ of 0.065$)$, becoming similar to those before the reaction.

It is worth mentioning that, since the optical adsorption, photothermal conversion, thermal conductivity and reaction capability of BCM layer-graphene on mica are all distinct from those of BCM layer-graphene on Cu(111), identical UV irradiation could afford a totally different dynamic intermediate state in the reversible reaction process. Upon an identical irradiation, the reaction yield obtained from the quasi-free-standing graphene on mica can thus be very different from that from the graphene- $\mathrm{Cu}(111)$. Importantly, these Raman results from the BCM layer on the quasi-free- 
standing graphene indicate that such a photocycloaddition can occur without the aid of a metal substrate, which is important in its real-life application for electronics.

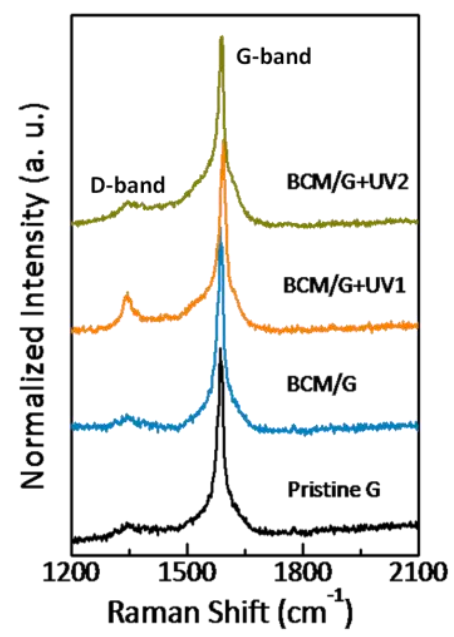

Supplementary Fig. 8| Raman spectra of the pristine quasi-free-standing graphene ('Pristine $\mathrm{G}^{\prime}$ ), BCM on the free-standing $\mathrm{G}$ without irradiation ('BCM/G'), BCM on the free-standing $\mathrm{G}$ after the first round $\mathrm{UV}$ irradiation ('BCM/G+UV1'), and $\mathrm{BCM}$ on the quasi-free-standing $\mathrm{G}$ after the second round UV irradiation ('BCM/G+UV2').

\section{Less robust $\mathrm{BCM}$ network on graphene- $\mathrm{Cu}(111)$ before the irradiation}
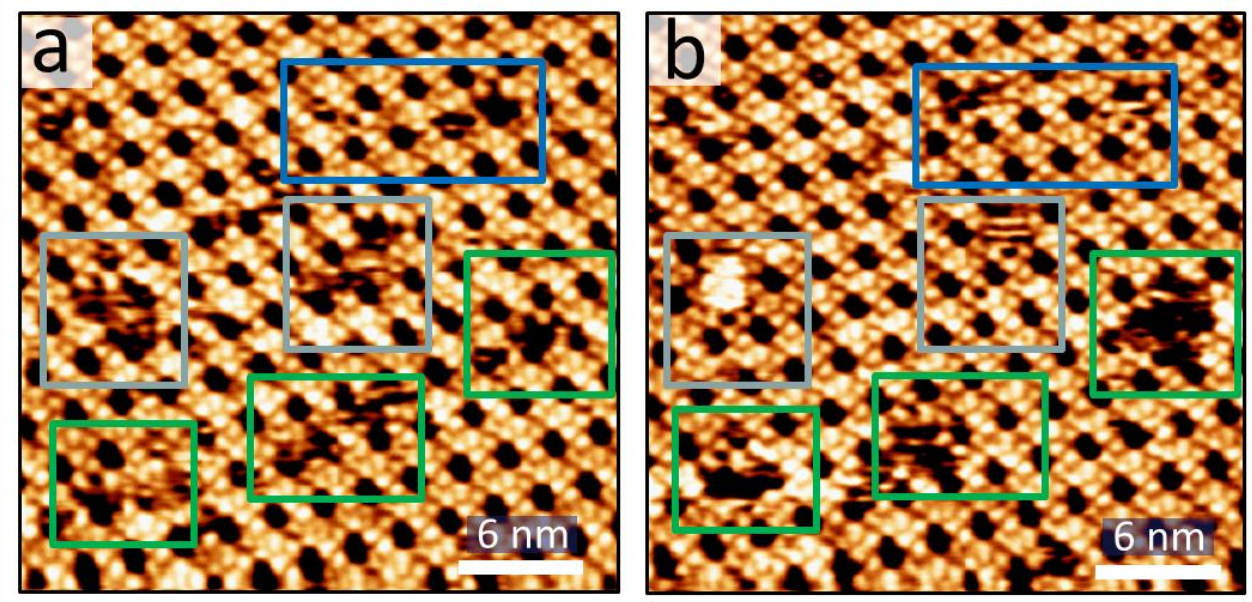

Supplementary Fig. 9| a-b, Sequential STM images of the BCM network on graphene-Cu(111) before irradiation, collected under the same scanning conditions $(-0.25 \mathrm{nA},-1250 \mathrm{mV})$. The images are from the same surface area. The green and blue rectangles point out the evident structural changes upon scanning. 
10. Remaining BCM network after desorption of the unreacted molecular zone.

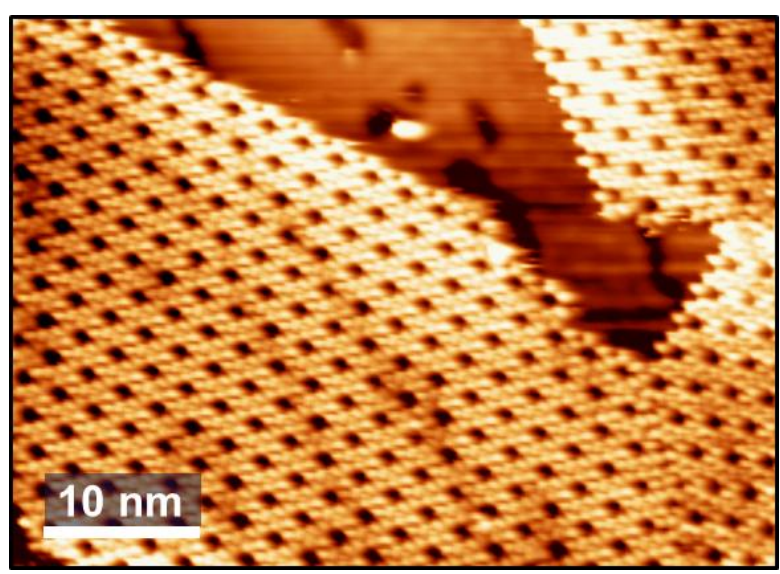

Supplementary Fig. 10| STM images of the UV-irradiated BCM network on graphene-Cu(111) after 10-min annealing at 376 K. 


\section{Stability of the excited state}

To estimate the stability of the covalently bonded configuration established after irradiation, a NEB simulation with 11 images including the initial (flat) and final (non-flat) configurations was performed using VASP. The calculated NEB band together with the images of the flat (on the left), non-flat (on the right) and the saddle point (in the middle) configurations are shown in Supplementary Fig. 11. One can see that the calculated barrier for the transition from the non-flat (excited) configuration back to the flat one is $0.77 \mathrm{eV}$, rendering the excited configuration being of sufficient stability.

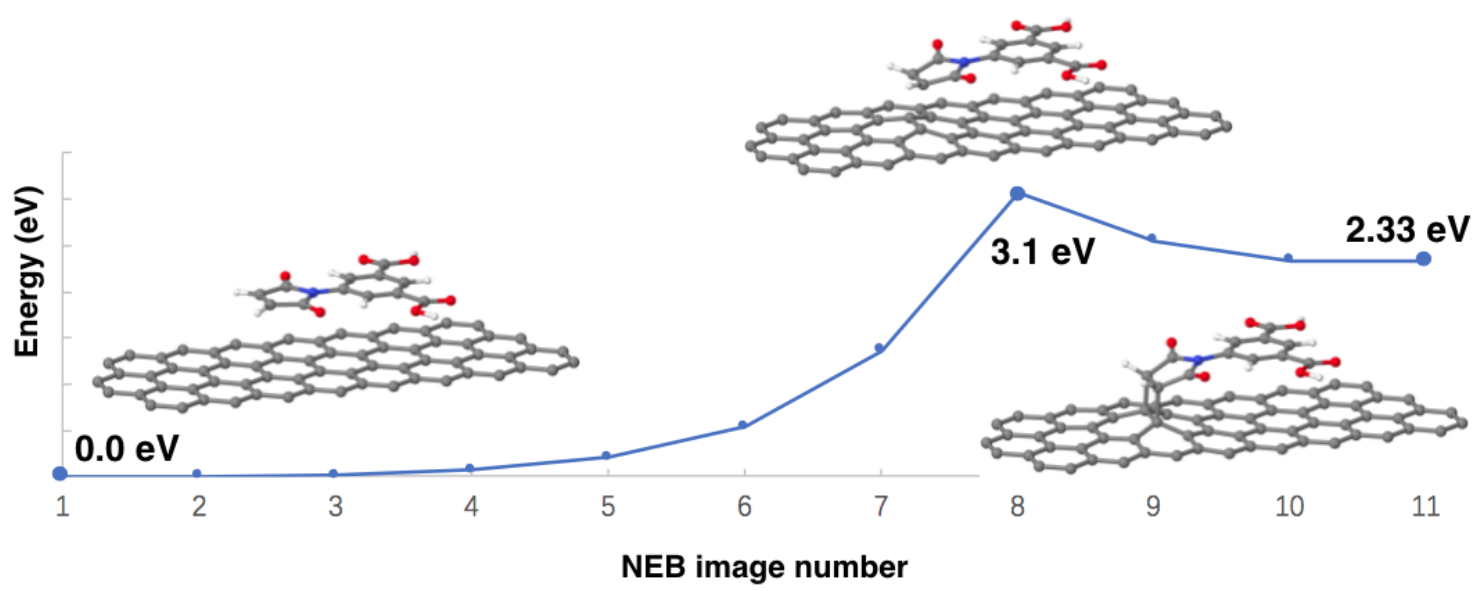

Supplementary Fig. 11| The calculated NEB band between the flat and non-flat configurations (as shown in Supplementary Fig. 7) for a single molecule on graphene. 


\section{Cycloaddition of a single $B C M$ at different sites of graphene on $\mathrm{Cu}(111)$}

We find that the obtained geometry of the BCM network above the underlying graphene favors only four molecules in each unit to have an appropriate angle and distance for covalent bonding with the $C$ atoms of the graphene layer, resulting in two $C 2$ and two $C 4$ cycloadditions, whilst $C 3$ cycloaddition does not occur. As summarized in Supplementary Table 2, the calculated energy increase of a single

$\mathrm{BCM}$ upon the cycloaddition $\Delta E=E_{\text {non-flat }}-E_{\text {flat }}\left(E_{\text {non-flat }}\right.$ is the energy of the final covalently bonded state and $E_{f l a t}$ is the energy of the initially physisorbed state) is calculated. Different cases of a single $\mathrm{BCM}$ in $\mathrm{C2}$ and $\mathrm{C4}$ cycloaddition with graphene-Cu(111) are considered, where the $\mathrm{C}$ atoms of the graphene layer positioned above (1) the fcc- and hcp-hollow sites of $\mathrm{Cu}(111)$; (2) the atop and hcphollow sites of $\mathrm{Cu}(111)$; (3) the atop and fcc-hollow sites of $\mathrm{Cu}(111)$, as depicted in Supplementary Fig. 12. A single BCM in $C 2$ and $C 4$ cycloaddition with free-standing graphene is also calculated.
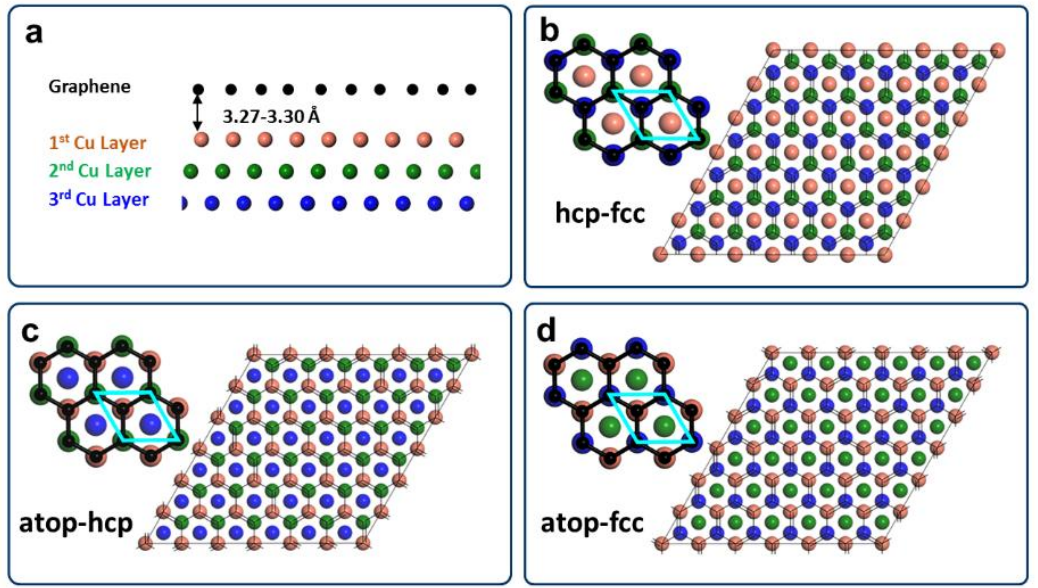
Supplementary Fig. 12 | a, Schematic illustration of the graphene sheet placed on top of three Cu layers of $\mathrm{Cu}(111)$, where the $\mathrm{C}$ atoms are in black, and $\mathrm{Cu}$ atoms in the $1^{\text {st }}, 2^{\text {nd }}$, and $3^{\text {rd }}$ layers are in bronze, green and blue, respectively. Schematic illustration of the graphene layer with the $\mathrm{C}$ atoms sitting above $\mathbf{b}$, fcc- and hcp-hollow ('fcc-hcp'), c, atop and hcp-hollow ('atop-hcp'), and d, atop and fcc-hollow ('atop-fcc') sites of $\mathrm{Cu}(111)$. The unit cell in each case is indicated by a cyan rhombus.

Supplementary Table 2. $\Delta E$ of a single BCM in $C 2$ and $C 4$ cycloaddition with the free-standing pristine graphene ('Pgraphene') and graphene- $\mathrm{Cu}(111)$.

\begin{tabular}{|c|c|c|}
\hline Energy (eV) & C2 & C4 \\
\hline fcc-hcp & $(2,3)$ & $(2,3)$ \\
\hline & 2.24 & 2.19 \\
\hline atop-hcp & $(1,2)$ & $(1,2)$ \\
\hline & 2.19 & 2.13 \\
\hline atop-fcc & $(1,3)$ & $(1,3)$ \\
\hline & 2.22 & 2.16 \\
\hline P-graphene & 2.18 & 2.11 \\
\hline
\end{tabular}

${ }^{*}(n, m)(n, m=1,2,3)$ the numbers correspond to the Cu layers atoms which are exactly beneath the graphene layer atoms.

\section{TD-DFT calculations}

Schematic illustration of an idealized process (indicated by the violet arrows) that activates the cycloaddition upon photoexcitation is shown in Supplementary Fig. 13. Note that the energy released due to transitions from higher to lower lying PES is transferred into atomic vibrations and hence must be responsible for the observed heating of the sample. A reverse process that restores the system back into the flat geometry upon the second-round irradiation works in a similar way, but starting with an excitation from the covalently-bonded non-flat form as the ground state back into the flat form.

Supplementary Table 3 contains the results of our TD-DFT calculations of the vertical singlet-tosinglet excitations of a single BCM molecule adsorbed on a free-standing graphene, for both the flat ("physisorbed") and non-flat ("covalently bonded") geometries. In both cases, strong oscillator strengths are obtained for transitions around $2.8 \mathrm{eV}$, which is within the applied energy region of the UV irradiation. The charge densities of the orbitals involved in some of the transitions are shown in Supplementary Fig. 14. 


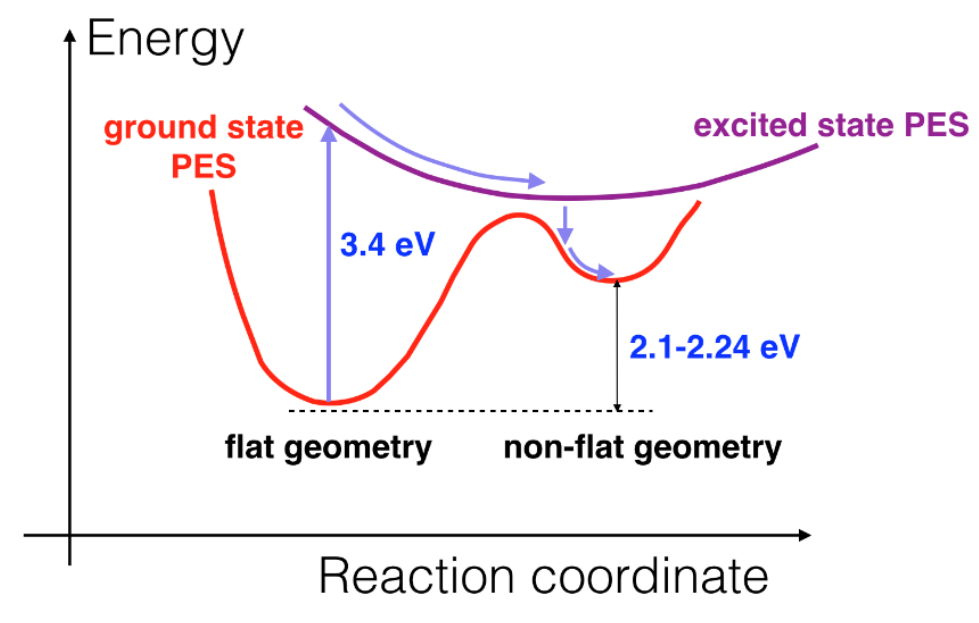

Supplementary Fig. 13| Schematic illustration of the irradiation process. Initially, the BCM molecule on graphene is in the flat geometry, which is the lowest energy minimum on the ground state potential energy surface (PES). Upon the photoexcitation of $3.4 \mathrm{eV}$, the molecule is promoted into a higher excited PES, where the system is first relaxed and then makes a transition (either radiative or non-radiative) back to the ground state PES. However, since the minimum of the excited state PES happens to be within the region of another energy well of the ground state PES, that is associated with the non-flat geometry of the molecule-graphene, the system stabilizes in this new state, i.e. in the cycloaddition configuration. The whole transition path is indicated with a set of violet arrows. This illustration has been greatly simplified; in reality, the molecule may go through a sequence of excited states down in energy after the excitation, before making its final transition into the cycloaddition state.

Supplementary Table 3. Comparison of the vertical singlet-to-singlet excitation energies of a single BCM molecule placed on graphene in the flat (physisorbed) and non-flat (covalently bonded) geometries. The letters $\mathrm{H}$ and $\mathrm{L}$ correspond to the HOMO and LUMO orbitals, respectively. In each case, all excitation states were calculated with the excitation energies of up to $4.0 \mathrm{eV}$. Oscillator strengths, which values are smaller than 1 a.u. for the flat geometry and 0.45 a.u. for the non-flat one, are not shown.

\begin{tabular}{cccc}
\hline Geometry & $\begin{array}{c}\text { Excitation Energy } \\
(\mathrm{eV})\end{array}$ & $\begin{array}{c}\text { Primary } \\
\text { Transition }\end{array}$ & $\begin{array}{c}\text { Oscillator Strength } \\
\text { (a.u.) }\end{array}$ \\
\hline \multirow{6}{*}{ Flat } & 2.01870 & $\mathrm{H}-1 \rightarrow \mathrm{L}+1$ & 1.35793 \\
& 2.02402 & $\mathrm{H} \rightarrow \mathrm{L}+3$ & 1.13691 \\
& 2.04489 & $\mathrm{H}-5 \rightarrow \mathrm{L}+5$ & 1.04766 \\
& 2.78697 & $\mathrm{H}-7 \rightarrow \mathrm{L}+7$ & 1.44582 \\
& 2.80534 & $\mathrm{H}-7 \rightarrow \mathrm{L}+8$ & 1.87383 \\
& 2.80980 & $\mathrm{H}-8 \rightarrow \mathrm{L}+8$ & 2.02534 \\
& 1.48258 & $\mathrm{H}-2 \rightarrow \mathrm{L}$ & 0.53954 \\
& 1.68345 & $\mathrm{H} \rightarrow \mathrm{L}+4$ & 0.75158 \\
Non-Flat & 2.04533 & $\mathrm{H}-4 \rightarrow \mathrm{L}+4$ & 0.46704 \\
& 2.81292 & $\mathrm{H}-10 \rightarrow \mathrm{L}+8$ & 0.49870 \\
& 2.82208 & $\mathrm{H}-9 \rightarrow \mathrm{L}+10$ & 0.75831 \\
& 2.82814 & $\mathrm{H}-8 \rightarrow \mathrm{L}+10$ & 0.88113 \\
\hline
\end{tabular}

${ }^{a}$ Occupied and unoccupied orbitals participating in the transitions are indicated, respectively, by counting from $\mathrm{H}$ (down) or $L$ (up). For instance, $\mathrm{H}-1$ is corresponding to the occupied orbital preceding the HOMO, while $\mathrm{L}+8$ is corresponding to the eights unoccupied orbital counting up from the LUMO. 

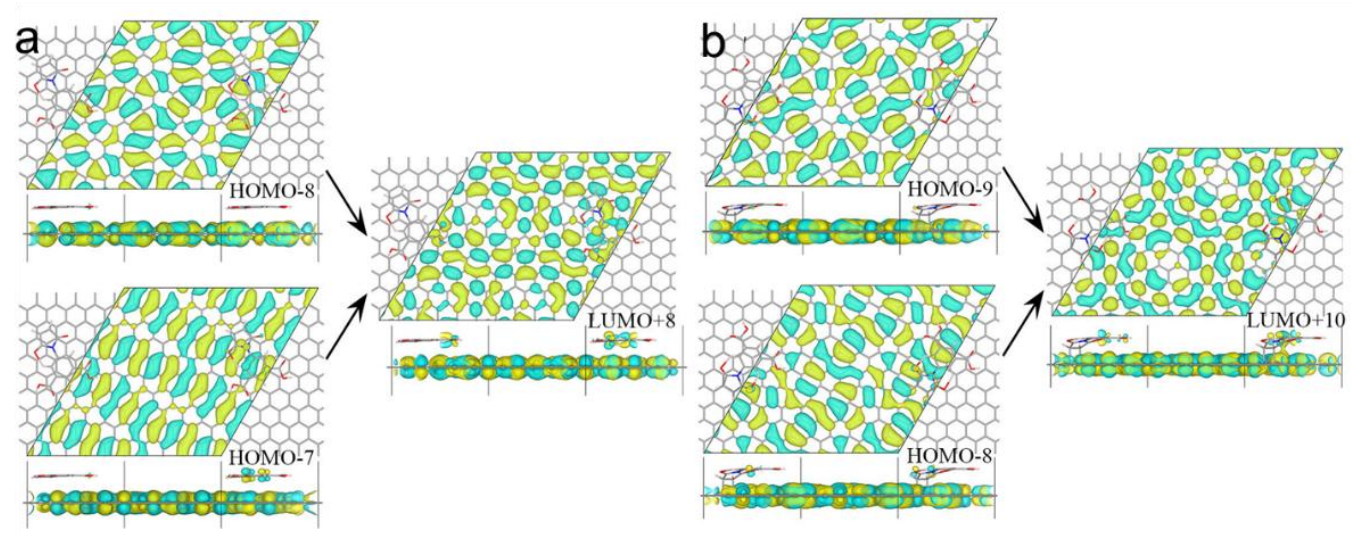

Supplementary Fig. 14| Orbitals involved in the primary transitions with the excitation energy of $2.8 \mathrm{eV}$ in the $\mathbf{a}$, flat and $\mathbf{b}$, non-flat geometries, shown in top- and side-views. Upon excitation, a charge transfer from the graphene sheet to the molecule is observed in both cases.

\section{Sample temperature elevation upon UV irradiation}

To monitor the sample temperature variation upon the UV irradiation, a K-type thermocouple was firmly attached to the side edge of the hat-shaped Cu substrate, avoiding immediate exposure to the irradiation. The temperature elevation plot was based on 10 independent measurements of the irradiation (Supplementary Fig. 15). In fact, it was rather challenging to measure the actual temperature on the sample surface: (1) the measurement accuracy in this temperature range is not very high; (2) the temperature (induced by the irradiation) at the side edge would be lower than that of the sample surface; (3) the absorbed photons from the irradiation would not instantly be dissipated. Due to the latter two reasons, the local temperature could be higher than that in the plot, and the temperature difference for the sample irradiated for 8 and 11 min could be also larger. However, it is certain that the temperature increased with the irradiation duration, whilst the temperature elevation rate decreased gradually. The temperature almost reached equilbium state after 25-min irradiation, which was around $319 \mathrm{~K}$. Considering the high thermal conductivity of $\mathrm{Cu}$ and the long equiblium time, this temperature of $319 \mathrm{~K}$ would not be very different from the actual surface temperature.

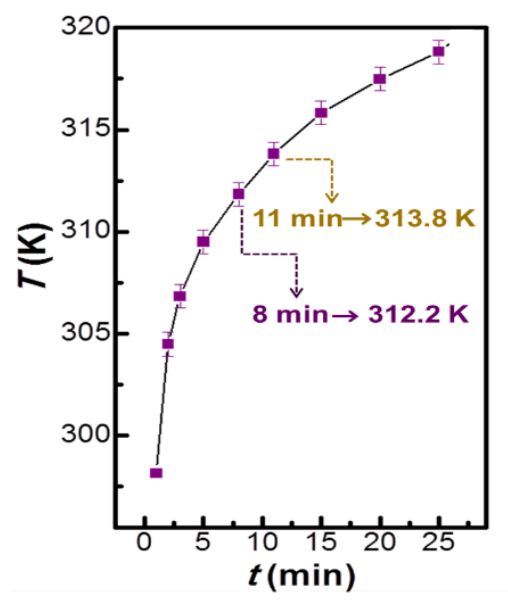

Supplementary Fig.15| Temperature elevation of the sample upon the UV irradiation. 


\section{References}

1. Cao, Y. et al. Diels-Alder reactions of graphene: computational predictions of products and sites of reaction. J. Am. Chem. Soc. 135, 17643-17649 (2013).

2. Denis, P. A. Organic chemistry of graphene: The Diels-Alder reaction. Chem.-Eur. J. 19, 1571915725 (2013). 